\title{
Processes and Procedures for Application of CFD to Nuclear Reactor Safety Analysis
}

Richard W. Johnson

Richard R. Schultz

Patrick J. Roache

Ismail B. Celik

William D. Pointer

Yassin A. Hassan

September 2006

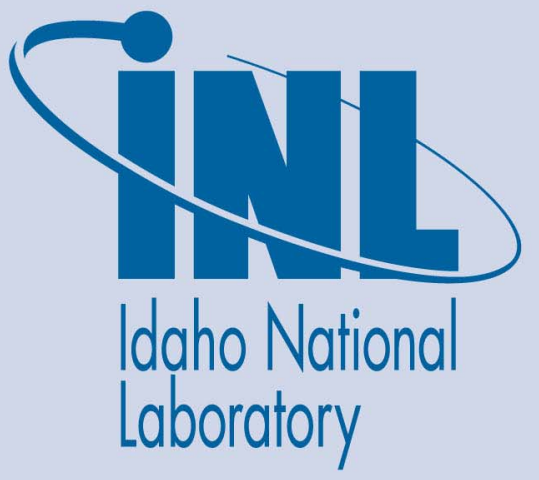

The INL is a U.S. Department of Energy National Laboratory operated by Battelle Energy Alliance 
INL/EXT-06-11789

\section{Processes and Procedures for Application of CFD to Nuclear Reactor Safety Analysis}

adaho National Laboratory

${ }^{\text {bSocorro, NM }}$

${ }^{c}$ University of West Virginia

${ }^{\mathrm{d}}$ Argonne National Laboratory

${ }^{\mathrm{e}}$ Texas A \& M University
Richard W. Johnson a

Richard R. Schultz ${ }^{a}$

Patrick J. Roache

Ismail B. Celik ${ }^{\mathrm{C}}$

William D. Pointer ${ }^{d}$

Yassin A. Hassan ${ }^{\mathrm{e}}$

September 2006
Idaho National Laboratory
Idaho Falls, Idaho 83415

Prepared for the

U.S. Department of Energy

Office of Nuclear Energy

Under DOE Idaho Operations Office

Contract DE-AC07-05ID14517 


\section{EXECUTIVE SUMMARY}

Traditionally, nuclear reactor safety analysis has been performed using systems analysis codes such as RELAP5, which was developed at the INL. However, goals established by the Generation IV program, especially the desire to increase efficiency, has lead to an increase in operating temperatures for the reactors. This increase pushes reactor materials to operate towards their upper temperature limits relative to structural integrity. Because there will be some finite variation of the power density in the reactor core, there will be a potential for local hot spots to occur in the reactor vessel. Hence, it has become apparent that detailed analysis will be required to ensure that local 'hot spots' do not exceed safety limits. It is generally accepted that computational fluid dynamics (CFD) codes are intrinsically capable of simulating fluid dynamics and heat transport locally because they are based on 'first principles.' Indeed, CFD analysis has reached a fairly mature level of development, including the commercial level. However, CFD experts are aware that even though commercial codes are capable of simulating local fluid and thermal physics, great care must be taken in their application to avoid errors caused by such things as inappropriate grid meshing, low-order discretization schemes, lack of iterative convergence and inaccurate time-stepping. Just as important is the choice of a turbulence model for turbulent flow simulation. Turbulence models model the effects of turbulent transport of mass, momentum and energy, but are not necessarily applicable for wide ranges of flow types. Therefore, there is a wellrecognized need to establish practices and procedures for the proper application of CFD to simulate flow physics accurately and establish the level of uncertainty of such computations.

The present document represents contributions of CFD experts on what the basic practices, procedures and guidelines should be to aid CFD analysts to obtain accurate estimates of the flow and energy transport as applied to nuclear reactor safety. However, it is expected that these practices and procedures will require updating from time to time as research and development affect them or replace them with better procedures. The practices and procedures are categorized into five groups. These are:

1. Code Verification

2. Code and Calculation Documentation

3. Reduction of Numerical Error

4. Quantification of Numerical Uncertainty (Calculation Verification)

5. Calculation Validation.

These five categories have been identified from procedures currently required of CFD simulations such as those required for publication of a paper in the ASME Journal of Fluids Engineering and from the literature such as Roache [1998]. Code verification refers to the demonstration that the equations of fluid and energy transport have been correctly coded in the CFD code. Code and calculation documentation simply means that the equations and their 
discretizations, etc., and boundary and initial conditions used to pose the fluid flow problem are fully described in available documentation. Reduction of numerical error refers to practices and procedures to lower numerical errors to negligible or very low levels as is reasonably possible (such as avoiding use of first-order discretizations). The quantification of numerical uncertainty is also known as calculation verification. This means that estimates are made of numerical error to allow the characterization of the numerical results with a certain confidence level. Numerical error in this case does not include error due to models such as turbulence models. Calculation validation is the process of comparing simulation results to experimental data to demonstrate level of agreement. Validation does include the effects of modeling errors as well as numerical and experimental errors.

A key issue in the validation process of numerical results is the existence of appropriate experimental data to use for validation purposes. It has become apparent, Roache [1998], that much of the experimental database for fluid flows is not adequate or has large errors associated with it. It has been recognized that appropriate validation data sets must meet some minimal set of criteria such as completeness and estimation of experimental uncertainty. Hence, the present report includes a section on experimental accuracy and guidelines for future experiments that will provide suitable validation data for CFD calculations.

Finally, whether the numerical analysis achieves some acceptance level is not addressed in these practices and procedures. Acceptance level, in fact, is a management or regulatory body determination based on their needs and requirements. The following sections will outline the current consensus on each of the above categories, including how the CFD analyst should or can address each one in order to ultimately produce a simulation that is characterized by an appropriate validation metric. The present report represents the current thinking and recommendations of the several authors as well as experts cited from the literature. It is expected that improvements and/or updates based on future research and investigation will be made to the guidelines herein presented. 


\section{CONTENTS}

EXECUTIVE SUMMARY

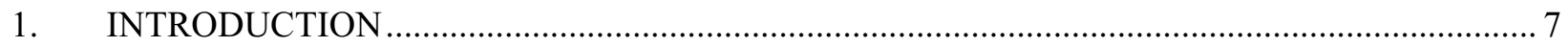

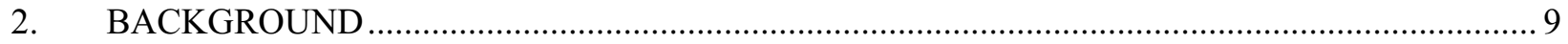

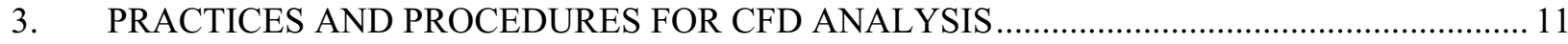

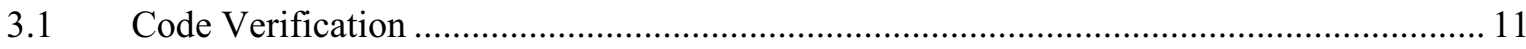

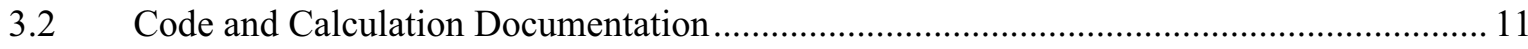

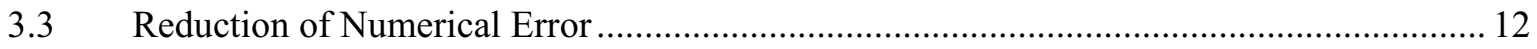

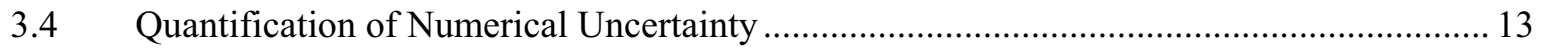

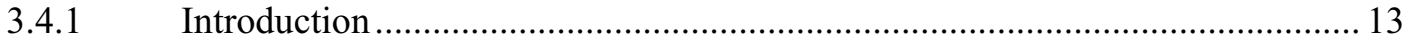

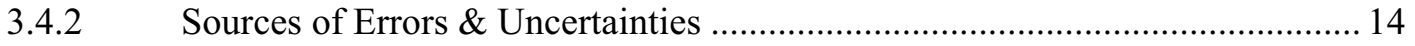

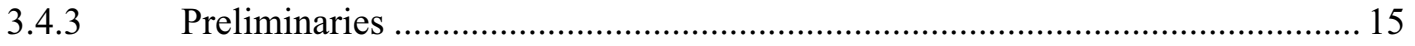

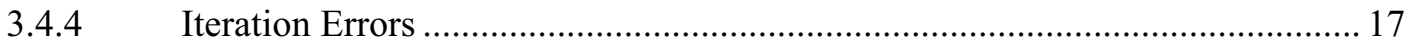

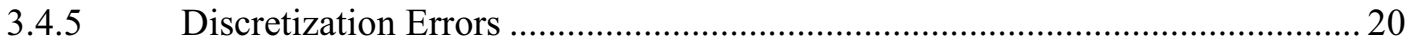

3.4.6 Numerical Uncertainty and Discretization Error Bars ................................... 25

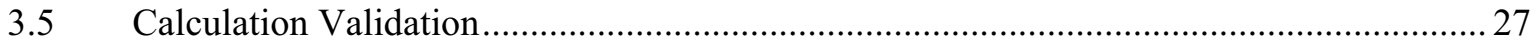

3.5.1 Method for Determination of Validation Level ............................................ 27

3.5.2 Data Reporting Requirements for Standard Problem Participants .................... 28

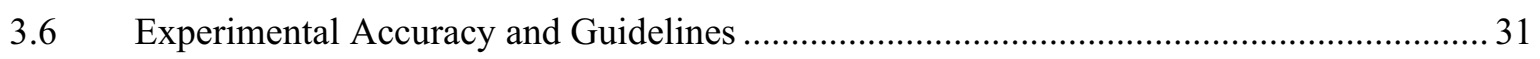

3.6.1 Velocity Measurement Techniques of the Standard Problems ........................... 33

3.6.2 Policy Statements on Experimental Accuracy ….......................................... 36

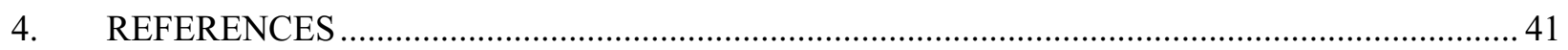

\section{FIGURES}

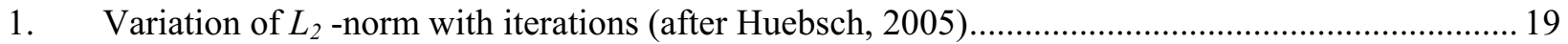

2. Comparison of solutions for iterative tolerances of $1 \times 10^{-3}$ to $1 \times 10^{-6}$ for (a) the mean stream-wise velocity $\mathrm{U}$, (b) the mean transverse velocity V, (c) the transverse normal stress $\overline{v^{2}}$ and (d) and the shear stress $\overline{u v}$. (courtesy of R.W. Johnson, 2005)

3. (a): Axial velocity profiles for a two-dimensional turbulent backward-facing-step flow calculation, Ref: Celik \& Karatekin [1997]; (b): Fine-grid solution, with discretization error bars computed using Eq. (15d). 
4. (a): Axial velocity profiles for a two-dimensional laminar backward-facing-step flow calculation, Ref: Celik \& Badeau [2005]; (b): Fine-grid solution, with discretization error bars computed using Eq. (15d)

5. Sample signal from numerical simulation

6. Three sample decompositions of the signal shown in Figure 5 into average, $\overline{\mathrm{u}}$, and fluctuating, $\mathrm{u}^{\prime}$, components. In (a), the average component has a constant value and all fluctuations are included in the fluctuation component. In (b), the primary periodic part of the fluctuation is included in the average component definition. In (c), the sum of the primary and secondary periodic parts of the fluctuations are included in the average component definition. All decompositions are valid applications of the Reynolds averaging methodology

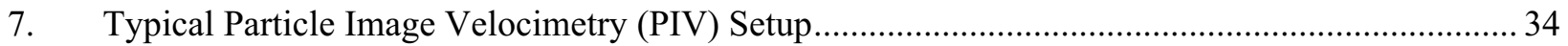

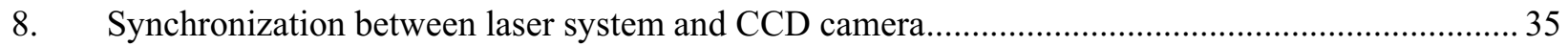

9. PIV and SPIV camera arrangement for 3-D two-phase bubbly flow measurements ...................... 36

\section{TABLE}

1. Sample calculations of discretization error . .25 


\section{Processes and Procedures for Application of CFD to Nuclear Reactor Safety Analysis}

\section{INTRODUCTION}

Traditionally, nuclear reactor safety analysis has been performed using systems analysis codes such as RELAP5, which was developed at the INL. However, goals established by the Generation IV program, especially the desire to increase efficiency, has lead to an increase in operating temperatures for the reactors. This increase pushes reactor materials to operate towards their upper temperature limits relative to structural integrity. Because there will be some finite variation of the power density in the reactor core, there will be a potential for local hot spots to occur in the reactor vessel. Hence, it has become apparent that detailed analysis will be required to ensure that local 'hot spots' do not exceed safety limits. It is generally accepted that computational fluid dynamics (CFD) codes are intrinsically capable of simulating fluid dynamics and heat transport locally because they are based on 'first principles.' Indeed, CFD analysis has reached a fairly mature level of development, including the commercial level. However, CFD experts are aware that even though commercial codes are capable of simulating local fluid and thermal physics, great care must be taken in their application to avoid errors caused by such things as inappropriate grid meshing, low-order discretization schemes, lack of iterative convergence and inaccurate time-stepping. Just as important is the choice of a turbulence model for turbulent flow simulation. Turbulence models model the effects of turbulent transport of mass, momentum and energy, but are not necessarily applicable for wide ranges of flow types. Therefore, there is a well-recognized need to establish practices and procedures for the proper application of CFD to simulate flow physics accurately and establish the level of uncertainty of such computations.

The present document represents contributions of CFD experts on what the basic practices, procedures and guidelines should be to aid CFD analysts to obtain accurate estimates of the flow and energy transport as applied to nuclear reactor safety. However, it is expected that these practices and procedures will require updating from time to time as research and development affect them or replace them with better procedures. The practices and procedures are categorized into five groups. These are:

1. Code Verification

2. Code and Calculation Documentation

3. Reduction of Numerical Error

4. Quantification of Numerical Uncertainty (Calculation Verification)

5. Calculation Validation.

These five categories have been identified from procedures currently required of CFD simulations such as those required for publication of a paper in the ASME Journal of Fluids Engineering and from the literature such as Roache [1998]. Code verification refers to the demonstration that the equations of fluid and energy transport have been correctly coded in the CFD code. Code and calculation documentation simply means that the equations and their discretizations, etc., and boundary and initial conditions used to pose the fluid flow problem are fully described in available documentation. Reduction of numerical error refers to practices and procedures to lower numerical errors to negligible or very low levels as is reasonably possible (such as avoiding use of first-order discretizations). The quantification of numerical uncertainty is also known as calculation verification. This means that estimates are made of numerical error to allow the characterization of the numerical results with a certain confidence level. Numerical 
error in this case does not include error due to models such as turbulence models. Calculation validation is the process of comparing simulation results to experimental data to demonstrate level of agreement. Validation does include the effects of modeling errors as well as numerical and experimental errors.

A key issue in the validation process of numerical results is the existence of appropriate experimental data to use for validation purposes. It has become apparent, Roache [1998], that much of the experimental database for fluid flows is not adequate or has large errors associated with it. It has been recognized that appropriate validation data sets must meet some minimal set of criteria such as completeness and estimation of experimental uncertainty. Hence, the present report includes a section on experimental accuracy and guidelines for future experiments that will provide suitable validation data for CFD calculations.

Finally, whether the numerical analysis achieves some acceptance level is not addressed in these practices and procedures. Acceptance level, in fact, is a management or regulatory body determination based on their needs and requirements. The following sections will outline the current consensus on each of the above categories, including how the CFD analyst should or can address each one in order to ultimately produce a simulation that is characterized by an appropriate validation metric. The present report represents the current thinking and recommendations of the several authors as well as experts cited from the literature. It is expected that improvements and/or updates based on future research and investigation will be made to the guidelines herein presented. 


\section{BACKGROUND}

Since the creation of the U.S. Nuclear Regulatory Commission, it has been required that electrical utilities desiring to build nuclear reactors to supply the public with electricity pass through a licensing process to demonstrate as well as possible that the reactor will operate safely and not pose a severe hazard to the public. The licensing process has typically included computer-based analysis to compute the reactor response to various accident scenarios as well as normal operation. Such analyses are typically performed using systems analysis codes, such as RELAP5. Nevertheless, such codes have been subjected to validation requirements to demonstrate their applicability and accuracy. In the case where a computational fluid dynamics code is used to perform safety analysis, it is expected that verification and validation of the CFD code used will also be subjected to validation requirements.

In the early years of the development of methods to numerically solve the equations of fluid dynamics, which are nonlinear, coupled, partial differential equations, most research performed was of interest to the CFD community and were accepted for publication in professional journals. However, after a level of maturation of CFD numerical methods, it began to become clear that some methods and practices usually yielded results with poor accuracy. An example of a method that usually produces poor accuracy is the two-point upwind scheme applied to the advection terms. Additionally, it became clear that certain practices would avoid or generally reduce numerical error if applied to CFD simulations. An example of a practice that reduces numerical error is to vary the coarseness/fineness of the grid to see where the calculations are grid independent and use the grid-independent results. Actually, an assessment of numerical accuracy or uncertainty cannot be made from one set of results obtained on one grid.

The first formal requirement for articles that reported numerical results for fluid flow submitted for review for publication in an archival journal was made by the Journal of Fluids Engineering (JFE), sponsored by the American Society of Mechanical Engineers (ASME), Roache [1999]. This statement reads as follows:

The Journal of Fluids Engineering will not accept for publication any paper reporting the numerical solution of a fluids engineering problem that fails to address the task of systematic truncation error testing and accuracy estimation.

Since then, other journals and organizations have added requirements for acceptance of submitted manuscripts, involving numerical and/or experimental results, or guidelines and practices to reduce numerical error and avoid major sources of inaccuracy. The Journal of Fluids Engineering has updated its requirements for the publication of papers containing numerical results. Current requirements are available on the ASME website ${ }^{f}$. The American Institute of Aeronautics and Astronautics (AIAA) has recently released a new set of guidelines for articles submitted to its journals for review for publication ${ }^{\mathrm{g}}$. The European Research Community on Flow, Turbulence and Combustion (ERCOFTAC) has published a large set of guidelines ${ }^{\mathrm{h}}$ for industrial users of CFD that addresses many issues of appropriate application of various models, etc. Many of these guidelines are covered in instruction manuals provided by commercial CFD code suppliers. It is not intended that the present guidelines will encompass such detail, but will be similar to the level of requirements established by the JFE. Finally, the European Union sponsored a project named 'Evaluation of Computational Fluid Dynamics Methods for Reactor Safety Analysis' (ECORA) ${ }^{i}$ that published some preliminary guidelines for the application of CFD to nuclear

\footnotetext{
${ }^{\mathrm{f}}$ http://journaltool.asme.org/Templates/JFENumAccuracy.pdf

${ }^{\mathrm{g}} \mathrm{http}: / /$ www.writetrack.net/aiaa/documents/NumericalAccuracy.pdf

${ }^{\mathrm{h}} \mathrm{http}: / /$ www.ercoftac.org/

${ }^{\mathrm{i}}$ http://domino.grs.de/ecora/ecora.nsf/
} 
safety analysis. The ECORA guidelines are subject to further development under the auspices of the international Nuclear Energy Agency (NEA).

The current requirements for papers containing numerical results and submitted for review to the ASME Journal of Fluids Engineering are called 'The Statement of Numerical Accuracy'. These requirements are:

Journal of Fluids Engineering Statement of Numerical Accuracy

1. The basic features of the method including formal truncation error of individual terms in the governing numerical equations must be described.

2. Methods must be at least second order accurate in space.

3. Inherent or artificial viscosity (or diffusivity) must be assessed and minimized.

4. Grid independence or convergence must be established.

5. When appropriate, iterative convergence must be addressed.

6. In transient calculations, phase error must be assessed and minimized.

7. The accuracy and implementation of boundary and initial conditions must be fully explained.

8. An existing code must be fully cited in easily available references.

9. Benchmark solutions may be used for validation for a specific class of problems.

10. Reliable experimental results may be used to validate a solution.

Of the above requirements, items 1,7 and 8 can be categorized as code and calculation documentation, items $2-6$ are practices and procedures to reduce numerical error and items $9 \& 10$ relate to calculation validation.

The present guidelines encompass the Journal of Fluids Engineering "Statement of Numerical Accuracy,' with some important additions. The additions include code verification and quantification of numerical uncertainty. The new AIAA "Editorial Policy Statement on Numerical and Experimental Accuracy," also includes a requirement for code verification.

The addition of a procedure to quantify numerical uncertainty is based on the NRC's historical desire to estimate uncertainty of traditional systems analysis codes used in reactor safety analysis. Though the quantification of numerical uncertainty is not currently required by the Journal of Fluids Engineering, an example of such a calculation based on Richardson extrapolation accompanies the JFE's "Statement on Numerical Accuracy." The guidelines are presented and discussed in the following section. 


\section{PRACTICES AND PROCEDURES FOR CFD ANALYSIS}

Practices and procedures are divided into several categories to indicate the goal and intent of each. These categories include Code Verification, Code and Calculation Documentation, Reduction of Numerical Error, Quantification of Numerical Uncertainty and Calculation Validation.

\subsection{Code Verification}

Code Verification involves the determination of coding correctness, Roache [1998], a process separate from Calculation Verification (the Quantification of Numerical Uncertainty). INL recognizes that the candidate CFD codes that will be applied to reactor safety analysis will already have been subjected to a variety of Code Verification tests. What will be required is documentation of these tests.

Only those tests that exercise the options used in the particular computations need to be documented. The tests as a suite must be designed to exercise all the terms in the governing partial differential equations. For example, it is not adequate to only test the code on linearly varying solutions such as planar Couette flow, since this solution does not exercise vertical convection terms and others. The most complete and convincing type of Code Verification test uses the Method of Manufactured Solutions or MMS, Roache [1998], but this will not be required. If MMS is not used, it will probably be necessary to use a suite of test problems to demonstrate code correctness. For all of these problems, the observed rate of discretization error convergence should be documented and compared to a theoretical value for the discretization algorithms employed. If it is not, then more stringent requirements will be enforced during Calculation Verification (see Section 3.5).

The Code Verification must also include some data on the effect of iterative convergence criteria on numerical results. (See Section 3.3 below for details.)

\subsection{Code and Calculation Documentation}

A CFD code that is used for nuclear reactor safety analysis must be described in detail in code documentation. Such documentation should include describing equations used and their discretization as well as the basics methods used to obtain a solution. The truncation error and its formal order or accuracy should be given. The code documentation must include all details of implementation of turbulence models used in calculations. The code documentation should be available for reference to reviewers who must review the associated CFD results.

Code Verification tests must be documented, as already noted.

For each CFD calculation performed and submitted as a safety analysis, assumptions must be listed along with the details of the methods and models used. Other details including, but not limited to boundary and initial conditions, model constants (parameters) and $\mathrm{y}^{+}$information must also be provided. Options not used in the calculation need not be documented.

Certain popular low-Re k- $\varepsilon$ turbulence models have recently been shown, with code exercises and analyses, to have a major problem with non-uniqueness, Rumsey et al [2006], Pettersson-Reif et al [2006]. These models sometimes generate non-unique solutions, with different solutions satisfying iterative convergence criteria dependent upon initial conditions, iterative solution algorithm and path, and possibly other equally extraneous factors. For obvious reasons, these models (see Rumsey et al [2006], Pettersson-Reif et al [2006]) will not be allowed. 


\subsection{Reduction of Numerical Error}

The reduction of numerical error is clearly a desirable objective for numerical calculations. Lessons have clearly been learned about what not to do when using CFD for numerical analysis. These have been canonized in the requirements for manuscripts submitted to well-known CFD journals. It therefore seems prudent to apply them to the application of CFD to reactor safety analysis.

The requirements from the JFE "Statement on Numerical Accuracy" that are aimed at the reduction of numerical error include:

- Methods must be at least second order accurate in space.

- Inherent or artificial viscosity (or diffusivity) must be assessed and minimized.

- $\quad$ Grid independence or convergence must be established.

- When appropriate, iterative convergence must be addressed.

- In transient calculations, phase error must be assessed and minimized.

It has been found that first-order errors from using first-order upwind discretization for the advection terms in the Navier-Stokes equations can be so large, that they swamp the true physics. The utility of using first-order upwinding may be to obtain a better initial guess to the correct solution than just using constant values. First-order upwinding should not be used for the final results. This guideline proscribes hybrid methods that use high-order schemes where possible and first-order schemes where necessary.

Inherent numerical viscosity is a result of truncation errors for even second-order accurate discretization schemes and represents numerical error. The natural approach to reduce these errors is to refine the numerical mesh to the point that it is negligible. Another approach that can be used is to use higher-order methods that eliminate some of the truncation error from the scheme. Artificial viscosity is typically numerical viscosity that is intentionally added to a numerical scheme to achieve convergence.

Grid independence is the process of refining the grid from some starting point until numerical results stop changing or change by negligible amounts. Theoretically, the results will continue to change until the grid spacing approach zero. The precision of the machine, however, will halt this process at a finite grid spacing. This is sometimes referred to achieving machine zero (of the residuals). Not only is the process of obtaining grid independence important to reducing numerical errors, it is also a good way to obtain estimated of numerical uncertainty, Section 3.4.

Iterative convergence relates to the number of iterations required to obtain residuals that are sufficiently close to zero either for a steady-state problem, or for each time step in an unsteady problem. This error is in addition to the numerical error associated with the truncation error terms. Because of the well-known and unacceptable sensitivity of some commercial codes to the iteration tolerance, and the too lax default tolerance, the final calculations must determine this effect. At least two levels of iteration tolerance must be shown and the sensitivity presented. For example, if results for a solution functional $f$ are presented using a default iteration tolerance of (say) $10^{-3}$ reduction in residual from the initial condition, as required in Freitas et al [2003], then another calculation with $10^{-4}$ will be required, and the sensitivity $f^{\prime}{ }_{10}$ will be stated as the normalized \% change in $f$ per decade of change in iteration tolerance. 
$f_{10}^{\prime}=\Delta f=\left[f\left(10^{-4}\right)-f\left(10^{-3}\right)\right] / f_{\text {norm }} \times 100 \%$

The normalization can be based on $f_{\text {norm }}=f\left(10^{-3}\right)$ when divides by near zero are not a problem, otherwise by another appropriate normalization. The final test of sufficient tightness of the iterative tolerance will be the acceptability of the final results based on estimation of numerical uncertainty and validation metrics.

For transient calculations, the same convergence criterion should apply as for spatial convergence (grid independence). The time step should be refined until negligible change is obtained. Also, though not required by the Journal of Fluids Engineering, it is recommended that the time-wise discretization scheme should be second-order accurate or better. This is in accord with the latest AIAA requirement.

While there are other practices to reduce numerical error, the above will constitute the required practices for rector safety analysis at the present time. Certainly, other practices that reduce numerical error are allowed and even encouraged.

\subsection{Quantification of Numerical Uncertainty}

\subsubsection{Introduction}

This report is prepared with the premise that the use of Computational Fluid Dynamics (CFD) in prediction of key reactor parameters such as maximum wall temperature, cladding temperature, pressure drop, etc., will improve the safety analysis for the Next Generation Nuclear Plants (NGNP); in particular, very high temperature (gas cooled) reactors (VHTR). Here, the term CFD is used in the context of a software package that is designed to solve the coupled, nonlinear partial differential equations governing fluid flow and heat transfer, including conduction, convection and radiation. Usually, these equation sets are referred to as the Navier-Stokes equations plus the energy equation. Since the focus of this report is primarily VHTR, we will consider only single component, single phase flows without chemical reactions. Being essentially a computer code or computational tool, many aspects of CFD uncertainty have been covered in the International Atomic Energy Agency (IAEA) document entitled "Uncertainty Evaluation in Best Estimate Safety Analysis for Nuclear Power Plants", (draft IAEA document), which will be referred, here and after, as the "IAEA Document."

The present document should be taken as an amendment to the IAEA Doc, not as a substitute. In this regard, frequent reference will be made to this document and aspects already covered will not be repeated unless deemed necessary for clarity. Furthermore, our recommendation of certain uncertainty evaluation methods also cited in the IAEA document will be biased more towards deterministic methods. This preference is based on our past experience and may be judged as an arbitrary decision by some others.

The objective of this current document is to provide guidance for evaluation, assessment and quantification of overall uncertainty in a CFD calculation pertaining to a standard problem identified by the Standard Problem Committee (SPC). In this regard, we shall address issues related to calculation verification.

In what follows, we identify various sources of uncertainty and errors, then present methodology and examples of how each of these can be quantified and recommend a procedure for quantifying the overall uncertainty in a critical system parameter which is the result of CFD calculation. We also provide guidelines for uniform reporting of verification and validation studies. The recommended guidelines in this document should not be viewed as rigid, unchangeable rules; rather, they should be viewed as best 
practice guidelines and recommendations that are subject to evolution as more robust and reliable methods become available.

\subsubsection{Sources of Errors \& Uncertainties}

A through list of the sources of uncertainties relevant to nuclear power plants (NPP) is listed and elaborated upon in the IAEA Document. These can be categorized into four major classes, namely uncertainties in

1. material properties (e.g., density, conductivity, porosity, emissivity, etc.)

2. geometrical dimensions (e.g., pipe diameter, plate thickness, surface roughness etc)

3. boundary and initial conditions, including size of the calculation domain (e.g. wall temperature, wall heat flux, inlet turbulent intensity and length scale)

4. numerical (e.g. arising from temporal and spatial discretization errors)

5. modeling, i.e. approximations and/or uncertainties in governing balance and constitutive equations)

The uncertain input parameters for a typical simulation may add up to hundreds of parameters. It is certainly not practical to run this many CFD calculations, especially when the phenomenon is threedimensional, and transient. Hence we recommend a slightly different approach than the usual practices that were outlined in the IAEA document. First of all, we do not consider model parameters such as those used in a certain turbulence model as arbitrary random parameters, instead we consider them as part of the model itself. The sensitivity analysis and optimization of the model parameters are usually performed (or should have been performed) during the development of that particular model.

Uncertainties in the material and geometrical parameters can be handled using any of the statistical methods given in the IAEA Document. For example, Wilks' formula (Wilks, 1941, 1942) can be used to determine the number of runs that would be required to establish two-sided (or one sided) statistical tolerance limits with $95 \%$ probability and confidence limits.

As for the inlet and boundary conditions, we recommend that expert judgment combined with sensitivity analysis of the most critical parameters that constitute the boundary and/or initial conditions for the simulation should be used to select specific boundary conditions and margin of variations in these conditions. It can be said that for a steady turbulent flow in an enclosure the most significant boundary conditions are the inlet conditions. Typically, inlet profiles of all three components of the velocity, turbulent kinetic energy, and the turbulence length scale at the inlet must be specified, in addition to the temperature (or enthalpy) profiles at the inlet. Reynolds stress models and algebraic stress models require all six components of the Reynolds stresses. For practical purposes, we recommend that the profile shapes and integrated flux of mass, momentum, energy, and turbulent kinetic energy be specified, and variations should be imposed on these for sensitivity analysis when one or two-equation turbulence models are used. The turbulence length scale (or equivalent information such as turbulent viscosity, turbulent dissipation rate etc) can be treated as derived quantities in the absence of more detailed experimental data. For higher order turbulence model, additional information needed should be derived from the same information provided for the two-equation turbulence models, unless such information is available from experiments. If boundary conditions are imposed at the outlet(s) and these boundary conditions are known to have upstream influence (e.g. in case of swirling flows pressure distribution at the outlet may have significant upstream influence when the swirl number is high), then these should be separately investigated.

Variations on the uncertain inlet flow conditions should be treated as part of the validation process (see 
Sec. 6). This essentially covers the domain size influence for internal flows. For external flows, boundary layers on curved surfaces, blades etc., with free stream turbulence, the extent of the calculation domain should be considered as an independent input parameter.

\subsubsection{Preliminaries}

\subsubsection{Reporting Requirements}

The computer code used for an application must be fully referenced, and previous verification studies must be briefly described. The word "verification" is used in this note in its broadest sense, meaning that the computer code is capable of solving a system of non-linear coupled partial differential equations with a properly posed set of initial and/or boundary conditions correctly, and reproduces the exact solution to these equations when sufficiently fine grid resolution (both in time and space) is employed. The formal order of accuracy in time and space for each equation solved should be also stated clearly, with proper references where this information is accessible to the readers. The governing equations, modeling assumptions, the constitutive equations, and all of the model parameters involved should be clearly defined and reported or appropriate references should be given where such information can be found easily.

\subsubsection{Definitions}

The scope of Computational Fluid Dynamics (CFD) may include a wide range of engineering problems such as combustion, multi-phase flow, radiation and thermal engineering among others. It may even include solid mechanics and solidification. The common ingredient in all of these applications is a mathematical model consisting of usually coupled non-linear partial differential equations (PDE's) with appropriately prescribed boundary and initial conditions. Let us denote such a model by the differential operator $L$ :

$L(\phi)=0$ with the boundary and initial conditions, respectively

$Q(\phi)=f(t)$ on the boundary $\Omega$ of the domain $\Theta$

$\phi(x, 0)=g(x)$ inside the domain $\Theta$

The equations are to be solved using some numerical method that calls for discretization of the equations and the boundary conditions using say finite differences on a mesh that can be represented by a grid parameter $h\left(x_{h}\right)$. The variable of interest $\phi$ is not only a function of space and time but also of some parameters such as turbulence model constants, species-reactions rates, and material properties, which usually contain some uncertainty.

The discretized counterparts of the above equations can be represented as

$$
\begin{aligned}
& L_{h}\left(\phi_{h}\right)=R_{h}\left(x_{h}, t_{h}, n_{i t e r}\right) \\
& Q_{h}\left(\phi_{h}\right)=f_{h}\left(t_{h}\right) \\
& \phi_{h}\left(x_{h}, 0\right)=g_{h}\left(x_{h}\right)
\end{aligned}
$$


Here, $\mathrm{n}_{\text {iter }}$ is the number of iterations, the subscript $\mathrm{h}$ denotes a numerical quantity calculated on a mesh represented by the grid parameter $h$ which is a function of cell volume and the time step. The r.h.s. of (2a) represents the residual after substituting the numerical solution into the discretized equations. This error may be due to incomplete convergence in temporal or spatial domain, round off errors, or simply due to accepting an approximate solution to start the next time step for example.

The numerical and modeling error, are defined respectively, by

$$
\begin{aligned}
& \varepsilon_{\text {num }}=\phi-\phi_{h}\left(x_{h}, n_{\text {iter }}\right) \\
& \varepsilon_{\text {mod }}=\phi_{\text {true }}-\phi
\end{aligned}
$$

Where $\phi_{\text {true }}$ is the "true" solution to the given physical problem that may be determined via experiments and/or direct numerical simulations. In many cases it is assumed that a unique true solution exits for a well defined physical problem. The solution error is, then, a combination of both modeling and numerical error, i.e.

$$
\varepsilon_{\text {sol }}=\phi_{\text {true }}-\phi_{h}=\varepsilon_{\text {num }}+\varepsilon_{\text {mod }}
$$

It is desired that the numerically calculated value $\phi_{h}$ tends to the 'unknown' exact solution, $\phi$, of the PDE (Eqs. 1a,b\&c) as h tends to zero, both locally and globally. This is what needs to be shown in a verification study. Such methods which are, at the same time, stable are classified as consistent methods. It is usually assumed in CFD applications that the numerical methods used are consistent. In the absence of any better alternative we shall also assume that this is the case in our applications provided that code being used is verified. Since the mesh size $h$ cannot be made infinitely small, there is always some discretization error in the numerical solution, among other errors such as iterative convergence errors and round off errors manifested as smearing and/or subtractive cancellation errors. Modeling errors, such as that arising from approximate physics imbedded into approximations of equations can be a significant source of error, which cannot be easily segregated from the numerical errors. A calculation of the error itself is not sufficient for quantification of 'solution uncertainty' in a statistical sense, but it is an essential ingredient in most uncertainty estimates. It should be noted that the sign of the numerical and modeling error can be opposite and error cancellation may occur resulting in a reduced solution error. errors, i.e.

By further assuming that numerical errors can be decomposed into discretization and iteration

$\varepsilon_{\text {num }}=\varepsilon_{\text {disc }}+\varepsilon_{\text {iter }}$

We write

$\varepsilon_{\text {sol }}=\phi_{\text {true }}-\phi_{h}=\varepsilon_{\text {disc }}+\varepsilon_{i t e r}+\varepsilon_{\text {mod }}$

Following the practice that is used in determining compounded experimental uncertainty (Stern et al. 2006; Coleman 1999) The overall uncertainty, $\delta_{\text {sol }}$, corresponding to the above mentioned errors in a CFD calculations can be written as

$\delta_{\text {sol }}^{2}=\delta_{\text {disc }}^{2}+\delta_{\text {iter }}^{2}+\delta_{\text {mod }}^{2}=\delta_{\text {mum }}^{2}+\delta_{\text {mod }}^{2}$ 
In what follows recommended procedures and examples are given for estimating the above mentioned errors and uncertainties.

\subsubsection{Iteration Errors}

The numerical solution of the non-linear coupled PDE's in CFD usually require iterative solutions. Iterations may be necessary for solution of linear system of equation, iterations to account for non-linear effects and iteration for sequential solution of the coupled PDE equations. In this document we refer to all errors arising from combinations of these iterations as "iteration errors."

Incomplete iteration errors can be a significant source of uncertainty in CFD applications (see for example Ferziger, 1989; Ferziger and Peric, 1996; Stern et al.2001, 2006b, Eça and Hoekstra 2006b, and others) Eca and Hoekstra have systematically investigated the effect of iteration error on the overall numerical uncertainty in solution of Reynolds-averaged Navier-Stokes equations. The numerical uncertainty considered in their paper is mostly related to the iteration and discretization errors in a nominally second order scheme in conjunction with the authors' own in house code. The discretization errors/uncertainty was also quantified using their own method based on Richardson extrapolation. As indicator of the iteration error the difference between consecutive iterations are considered as well as the normalized residuals of the discretization errors. They further considered an alternative indicator that is expressed as a power law i.e. error $=\alpha n^{q}$ where $n$ is the number of iterations and $\alpha$ and $q$ are undetermined parameters. These unknown parameters were determined using least squares curve fit.

After applications to several test cases and a meticulous analysis they reach the following conclusions:

- $\quad \mathrm{L}_{\infty}$ norm is the most appropriate norm to be used in such uncertainty assessment.

- The uncertainty measures based on error estimation from least square extrapolation were most reliable.

- If more conservatism is desired the standard deviation of the fit should be added to the uncertainty.

A more interesting conclusion is that the discretization errors (hence the uncertainty) are not independent of the iteration error. If the errors are of the same order of magnitude one can not simply add each component to reach a total uncertainly.

In the light of this observation it is most desirable to reduce the iteration error to at least 2-3 order of magnitude lower than the discretization error in CFD applications, so that the influence of incomplete iteration convergence on the over all uncertainty is minimized. If this desired convergence cannot be accomplished under special circumstances (for example, in case of positive scalars such as enthalpy, density, eddy viscosity, or dissipation rate, convergence to desired small residuals may not be possible) iteration convergence errors must also be quantified. For this purpose the following procedure is recommended.

Following Ferziger (1989 and 1996) the iteration error should be estimated by

$$
\varepsilon_{i t e r, i}^{n} \cong \frac{\left(\phi_{i}^{n+1}-\phi_{i}^{n}\right)}{\lambda_{i}-1}
$$


Where $\lambda_{1}$ is the principal eigen value of the solution matrix of the linear system, and it can be approximated from

$\lambda_{i} \cong \frac{\left\|\phi_{i}^{n+1}-\phi_{i}^{n}\right\|}{\left\|\phi_{i}^{n}-\phi_{i}^{n-1}\right\|}$

The uncertainty in iteration convergence can then be estimated as

$\delta_{\text {iter }} \cong \frac{\left\|\varepsilon_{\text {iter }, i}^{n}\right\|}{\lambda_{\text {ave }}-1}$

|| || being an appropriate norm, e.g., $L_{\infty}$ norm. Here $\lambda_{\text {ave }}$ is the average value of $\lambda_{1}$ over a reasonable number of iterations, if $\lambda_{\text {ave }} \approx 1.0$ the difference between two consecutive iterations would not be a good indicator of iteration error.

An alternative iterative error indicator is the scaled (normalized) residual of the discretized equation defined by

$$
\mathrm{R}_{\mathrm{h}}=\frac{\sum_{\text {cellsp }} \sum_{\mathrm{nb}}\left|\left(\mathrm{a}_{\mathrm{nb}} \phi_{\mathrm{nb}}+\mathrm{b}-\mathrm{a}_{\mathrm{p}} \phi_{\mathrm{p}}\right)\right|}{\sum_{\text {cellsp }}\left|\mathrm{a}_{\mathrm{p}} \phi_{\mathrm{p}}\right|}
$$

Here $a_{p}$ is the center coefficient of the discretization equation, $a_{n b}$ are the influence coefficients for the neighboring cells, and $b$ is the contribution of the source term. Correspondingly, $\phi_{p}$ is the value for a generic variable at the center cell and $\phi_{\mathrm{nb}}$ represent the ones at the center of the neighboring cells. Other properly defined normalized residuals may be used so long as they are fully documented and reported.

The iteration error indicator is taken as some norm over all the computational nodes of either the iteration error given by Eq (4a) or the normalized residual given by Eq. (5). Eça and Hoekstra (2006) recommend the $\mathrm{L} \infty$ norm. We recommend that the $\mathrm{L}_{\infty}$ norm of the errors defined by Eqs 4 and 5 be reported over a significantly large portion of the iterations (say more than 20\%) and convergence rates be shown graphically for each calculation to demonstrate iterative convergence, and numerical values be reported at the end of the iteration cycle for each time step for the slowest converging variable with the largest residual error.

A typical example is shown in Figure 1 (Huebsch, 2005). Here the $L_{2}$ norm of $\varepsilon_{\text {iter }}^{n}$ over the whole calculation domain is used as indicator of iterative convergence. Convergence is assumed to be good if $\left\|\varepsilon_{i t t r}\right\|$ is reduced by 3 to 4 orders of magnitude over each time step in unsteady calculations. Specifically

$L_{2}=\frac{\left[\sum_{i=1}^{n}\left\{\left(\Delta \Psi_{i}\right)^{2}+\left(\Delta \Omega_{i}\right)^{2}\right\}\right]^{\frac{1}{2}}}{\left(2 N_{x} N_{y}\right)^{1 / 2}}$ 
The 2D, pseudo-DNS calculations by Huebsch (2005) for an air foil with a step roughness at the surface illustrate the nature of iteration convergence as the grid is refined. The $L_{2}$-norm given by Eq. (5a) with $N_{x}=1001$ and $N_{x}=201$, plotted against iteration number in Figure 1, shows at least three orders of magnitude reduction for every time step. As the grid is refined the convergence get slower, as expected.

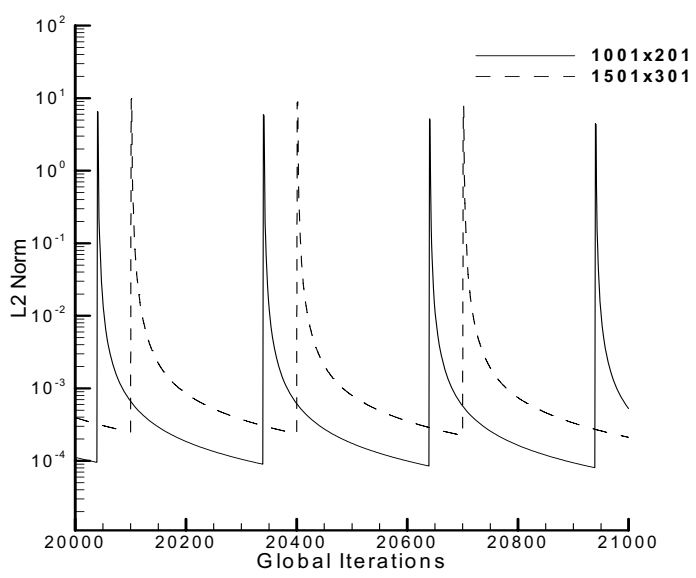

Figure 1. Variation of $L_{2}$-norm with iterations (after Huebsch, 2005).

Johnson (2005) has also demonstrated that incomplete iteration convergence may have significant influence on the steady state-solution for the flow past a staggered tube bundle (Simonin and Barcouda, 1986). Figure 2 shows an example calculation from Johnson (2005). The flow is actually unsteady and requires an unsteady calculation. The boundary conditions are periodic on all sides of the flow (left to right, top to bottom). The FLUENT 6.2.16 version of the Reynolds Stress Model is used in the calculations along with standard wall functions. The overall flow rate was matched to the data of Simonin and Barcouda by integrating the flow rate from the data. The $y+$ values for the walls were less than five.

Figures $2 \mathrm{a}$ and $2 \mathrm{c}$ are particularly interesting as they both show good agreement for results that are not iteratively converged.

It is recommended that, before any discretization error estimation is calculated, it must be ensured that iterative convergence (if iterative methods are used) is achieved with at least three orders of magnitude decrease in the normalized residuals for each equation solved and the iteration uncertainty is estimated from Eq. 4c. For time-dependent problems, iterative convergence at every time step should be checked, and sample convergence trends (see Figure 1) should be documented for selected, critically important, variables. Moreover, a posteriori analysis should follow after the computation of discretization errors to ensure that the iteration error is at least two orders of magnitude less than the discretization error. Hence it can be safely assumed that the iteration error uncertainty is negligible compared to discretization uncertainty; $\delta_{\text {iter }}<<\delta_{\text {disc }}$. 


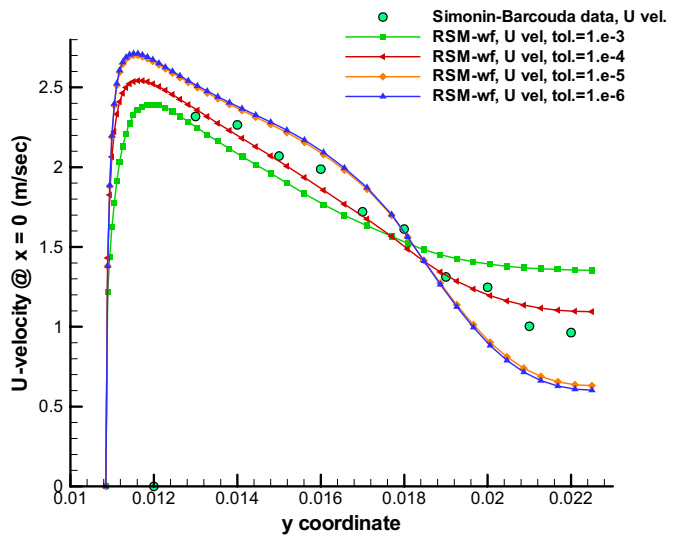

(a)

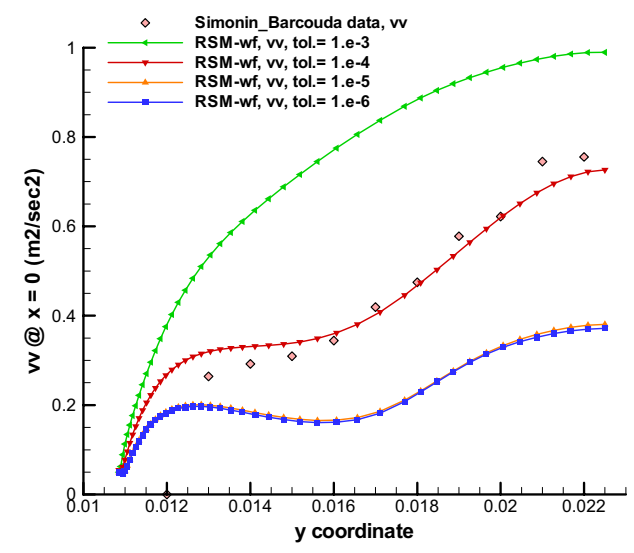

(c)

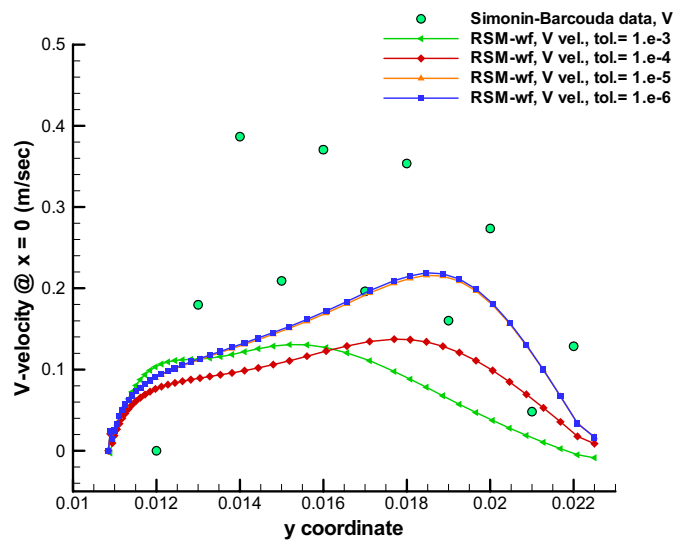

(b)

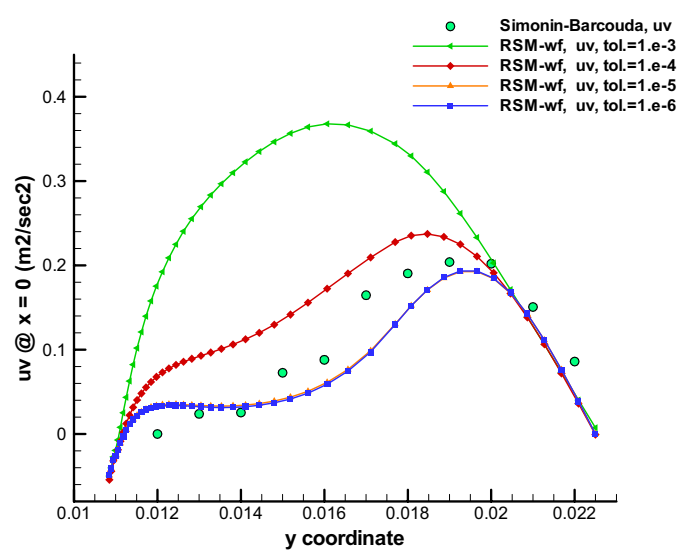

(d)

Figure 2. Comparison of solutions for iterative tolerances of $1 \times 10^{-3}$ to $1 \times 10^{-6}$ for (a) the mean streamwise velocity $\mathrm{U}$, (b) the mean transverse velocity $\mathrm{V}$, (c) the transverse normal stress $\overline{v^{2}}$ and (d) and the shear stress $\overline{u v}$. (courtesy of R.W. Johnson, 2005).

\subsubsection{Discretization Errors}

The recommended method for discretization error estimation is the Richardson extrapolation (RE) method (Richardson 1910, 1927). This method has been studied by many authors. Its intricacies, shortcomings and generalization have been widely investigated. A short list of references given in the bibliography (Celik, et al 1993,1997; Eça and Hoekstra 2002, 2004a, 2004b; Freitas 1993; Roache 1993, 1997, 1998; Stern, et al 2001, 2006) is selected for the direct relevance of these references to the subject. The RE method is far from being perfect. The local RE values of predicted variables may not exhibit a smooth, monotonic dependence on grid resolution, and in a time-dependent calculation, this non-smooth response will also be a function of time and space. Nonetheless, it is currently the most robust method available for the prediction of numerical uncertainty. Prospective users can find many examples in the above references. As new and more reliable methods emerge, the present document should be re-assessed and modified as needed. In particular, the RE based methods prescribed by Stern, et al. (2006), Eça and Hoekstra (2002, 2004a, 2006a), Celik and Li (2005) and Elizalde-Blancas and Celik (2006), Elizalde-Blancas et al. (2006) are recommended to be reviewed in the near future. Here we briefly present the main features of Richardson extrapolation. This method is based on a Taylor series expansion of the numerical solution $\phi_{h}\left(x_{h} ; h\right)$. 
$\phi_{h}=\phi_{0}+\sum_{k=1}^{\infty} C_{k}\left(x_{h}\right) h^{k}$

where

$C_{k}\left(x_{h}\right)=\left(\frac{\partial \phi_{h}}{\partial h}\right)_{h=0}$

It is possible to use four independent variables, $\Delta x, \Delta y, \Delta z$, and $\Delta t$ representing mesh size in the $\mathrm{x}-, \mathrm{y}-, \mathrm{z}-$ directions and in time, respectively but for brevity a single length scale $h$ is used to represent the grid in functional form

$h=\left(\Delta x \Delta y \Delta z \Delta x_{t}\right)^{1 / 4}$

where $\Delta x_{t}=u_{c h} \Delta x, \mathrm{u}_{\mathrm{ch}}$ being a characteristic velocity.

For steady flow calculations on structured grids a local mesh parameter can be defined by

$h=(\Delta x \Delta y \Delta z)^{1 / 3}$

for unstructured grids a reasonable global mesh parameter is

$$
\bar{h}=\left(\frac{\forall}{N}\right)^{1 / 3}
$$

where $\forall$ is the volume of the computational domain and $N$ is the number of computational cells; the corresponding local parameter can be calculated using the local cell volume. Equation (7b) indicates that $\mathrm{h}$ tends to zero as the grid number in one direction becomes large. This is problematic especially if $\Delta t$ is included in Eq. (7b). This can be avoided by defining the grid parameter, say by

$$
h=\left(\Delta x^{2}+\Delta y^{2}+\Delta z^{2}+\Delta t^{2}\right)^{1 / 2}
$$

However, equation (7d) yields multi-valued functions if the convergence trend changes for small $\Delta \mathrm{t}$. For unsteady RANS calculations $\Delta t$ is treated just as another mesh parameter (see Roache 1998, Celik and $\mathrm{Hu}$ 2004, Orozco et al. 2004, Celik et al. 2006b). The extrapolated value $\phi_{\text {ext }}$ is obtained from either

$\phi_{h}=\phi_{e x t}+c h^{p}$

or

$\phi_{h}=\phi_{e x t}+c_{1} h+c_{2} h^{2}$ 
using three numerical values of $\phi$ calculated on three significantly different grids. Equations (8a\&b) are valid in the asymptotic range where $h$ is sufficiently small so as to make the leading one or two terms in Eq. (6a) dominant. Equation $8 \mathrm{~b}$ is naturally valid only for schemes with nominally second order accuracy. However, since Eq. (8b) is essentially a curve fit, higher order methods will be represented by adjustment of the coefficients $c_{1}$ and $c_{2}$. When Eq. (8a) is used, usually, the locally calculated p values are averaged and then used in the extrapolation step. The extrapolation can also be performed using cubic splines either on the calculated quantity or on the approximate error i.e. the difference between the two consecutive grid solutions. These would be denoted as CS (cubic splines) and AES (approximate error splines) methods, respectively (see Celik and Li 2005 and Celik et al. 2005 for details). When utilizing Eq. (8a) we prefer the method suggested by Celik and Karatekin 1997 which even with three grid calculations partially accounts for non-monotonic convergence. Here, we briefly present some selected uncertainty estimates based on RE.

The Grid Convergence Index (GCI) proposed by Roache (1993) can be written as

$$
G C I_{f}=1.25\left|\frac{\phi_{e x t}-\phi_{f}}{\phi_{f}}\right|
$$

Here $\phi_{f}$ denotes the numerical solution on the fine grid. The extrapolated relative error (ERE) suggested by Celik \& Karatekin (1997) is given by

$$
E R E_{f}=\left|\frac{\phi_{e x t}-\phi_{f}}{\phi_{e x t}}\right|
$$

When the least squares method (as suggested by Eça and Hoekstra 2004a) is applied with more than 3 grids, the scatter in the data indicates a certain amount of uncertainty for the extrapolated value, which could be estimated by calculating the coefficient of variation (Celik and Li 2005). The coefficient of variation $(\mathrm{CV})$ for the least squares method (using at least 4 grids) is computed from

$$
\begin{aligned}
& \sigma_{r}^{2}=\sum_{i=1}^{n}\left[\phi_{i}-\left(\phi_{e x t}+\alpha h_{i}^{p}\right)\right]^{2} \\
& \sigma_{\phi / h}=\sqrt{\sigma_{r}^{2} / n} \\
& C V=\left|\frac{\sigma_{\phi / h}}{\phi_{e x t}}\right| \\
& E R E_{C V}=E R E_{f}+C V
\end{aligned}
$$

It should be noted that the least squares extrapolation treats each calculation on a different grid as if the error is a random variable which is contrary to the nature of the deterministic calculation being considered. Moreover, this method is only applicable with at least four calculations on four different grids. Being aware of the difficulty of this many calculations in industrial applications of CFD, we recommend RE on 3-grid calculations. 


\subsubsection{Procedure for Three Grid Calculations}

For three grid calculations, we recommend essentially the same procedure (with minor changes) as that recommended for the ASME Journal of Fluid Engineering (JFE) publication ${ }^{\mathrm{j}}$ ). This procedure involves the following five steps:

$\underline{\text { Step } 1}$ Define a representative cell, mesh or grid size $h$. For example for three-dimensional calculations

$$
h=\left[\frac{1}{N} \sum_{i=1}^{N}\left(\Delta V_{i}\right)\right]^{1 / 3}
$$

For two-dimensions,

$$
h=\left[\frac{1}{N} \sum_{i=1}^{N}\left(\Delta A_{i}\right)\right]^{1 / 2}
$$

where $\Delta V_{i}$ is the volume and $\Delta A_{i}$ is the area of the $i^{\text {th }}$ cell, and $N$ is the total number of cells used for the computations. Eqs. (12a and 12b) are to be used when integral quantities, e.g., drag coefficient, are considered. For field variables, the local cell size can be used. Clearly, if an observed global variable is used, it is then appropriate to use also an average "global" cell size.

$\underline{\text { Step } 2}$ Select three significantly different grids, and run simulations to determine the values of key variables important to the objective of the simulation study, for example, a variable $\phi$ critical to the conclusions being reported. It is desirable that the grid refinement factor, $r=h_{\text {coarse }} / h_{\text {fine }}$, be greater than 1.3. This value of 1.3 is based on experience, and not on formal derivation. The grid refinement should, however, be done systematically, that is, the refinement itself should be structured even if the grid is unstructured. Use of geometrically similar cells are preferable. Clearly the theory does not apply when selected local grid refinements are made with no refinement $(r=1)$ in other regions.

$\underline{\text { Step } 3}$ Let $\mathrm{h}_{1}<\mathrm{h}_{2}<\mathrm{h}_{3}$ and $\mathrm{r}_{21}=\mathrm{h}_{2} / \mathrm{h}_{1}, \mathrm{r}_{32}=\mathrm{h}_{3} / \mathrm{h}_{2}$, and calculate the apparent order, $\mathrm{p}$, of the method using the expression

$$
\begin{aligned}
& p=\frac{1}{\ln \left(r_{21}\right)}|\ln | \varepsilon_{32} / \varepsilon_{21}|+q(p)| \\
& q(p)=\ln \left(\frac{r_{21}^{p}-s}{r_{32}^{p}-s}\right) \\
& s=1 \cdot \operatorname{sign}\left(\varepsilon_{32} / \varepsilon_{21}\right)
\end{aligned}
$$

where $\varepsilon_{32}=\phi_{3}-\phi_{2}, \varepsilon_{21}=\phi_{2}-\phi_{1}, \phi_{k}$ denoting the solution on the $k^{\text {th }}$ grid. Note that $q(p)=0$ for $r=$ const. Eq. (13) can be solved using fixed-point iteration, with the initial guess equal to the first term. The absolute value in Eq. (13a) is necessary to ensure extrapolation towards $h=0$ (Celik \& Karatekin 1997). Negative values of $\varepsilon_{32} / \varepsilon_{21}<0$ are an indication of oscillatory convergence. If possible, the percentage

\footnotetext{
${ }^{\mathrm{j}}$ http://journaltool.asme.org/Templates/JFENumAccuracy.pdf
} 
occurrence of oscillatory convergence should also be reported. The agreement of the observed (or calculated) apparent order with the formal order of the scheme being used can be taken as a good indication of the grids being in the asymptotic range; the converse should not necessarily be taken as a sign of unsatisfactory calculations. It should be noted that if either $\varepsilon_{32}=a b s\left(\phi_{3^{-}} \phi_{2}\right)$ or $\varepsilon_{21}=a b s\left(\phi_{2^{-}} \phi_{1}\right)$ is "close" to zero, the above procedure does not work. This might be an indication of oscillatory convergence or, in rare situations, it may indicate that the "exact" solution has been attained. In such cases, if possible, calculations with additional grid refinement should be performed; if not, the results may be reported as such, with $\max \left(\varepsilon_{32}, \varepsilon_{21}, \varepsilon_{31}=a b s\left(\phi_{3^{-}} \phi_{1}\right)\right)$ being an indicator of the discretization error.

Step 4 Calculate the extrapolated values from

$$
\phi_{e x t}^{21}=\left(r_{21}^{p} \phi_{1}-\phi_{2}\right) /\left(r_{21}^{p}-1\right)
$$

Similarly, calculate $\phi_{\text {ext }}^{32}$.

$\underline{\text { Step } 5}$ Calculate and report the following error estimates, along with the apparent order $p$ :

Approximate relative error:

$$
\begin{aligned}
& e_{a}^{32}=\left|\frac{\phi_{2}-\phi_{3}}{\phi_{2}}\right| \\
& e_{a}^{21}=\left|\frac{\phi_{1}-\phi_{2}}{\phi_{1}}\right|
\end{aligned}
$$

Extrapolated relative error:

$$
e_{e x t}^{21}=\left|\frac{\phi_{e x t}^{12}-\phi_{1}}{\phi_{e x t}^{12}}\right|
$$

The fine grid convergence index:

$$
\mathrm{GCI}_{\text {fine }}^{21}=\frac{F_{s} e_{a}^{21}}{r_{21}^{p}-1}, \mathrm{~F}_{\mathrm{s}}=1.25
$$

Table 1 illustrates this calculation procedure for three selected grids. The data used are taken from Celik \& Karatekin 1997), where the turbulent two-dimensional flow over a backward facing step was simulated on non-uniform structured grids with total number of cells $N_{l}, N_{2}$, and $N_{3}$. Hence, according to Table 1, the numerical uncertainty in the fine-grid solution for the reattachment length should be reported as $2.2 \%$; for the axial velocity at the points (Table 1 ) as $1.1 \%$ and $0.5 \%$, respectively; note this does not account for modeling errors. 
Table 1. Sample calculations of discretization error.

\begin{tabular}{|l|l|l|l|}
\hline \multicolumn{1}{|c|}{$\begin{array}{c}\phi=\text { dimensionless } \\
\text { reattachment length } \\
\text { (with monotonic } \\
\text { convergence) }\end{array}$} & \multicolumn{1}{|c|}{$\begin{array}{c}\phi=\text { axial velocity at } \mathrm{x} / \mathrm{H}=8, \\
\mathrm{y}=0.0526 \\
(\mathrm{p}<1)\end{array}$} & $\begin{array}{c}\phi=\text { axial velocity at } \\
\mathrm{x} / \mathrm{H}=8, \mathrm{y}=0.0526 \\
\text { (with oscillatory } \\
\text { convergence) }\end{array}$ \\
\hline $\mathrm{N}_{1}, \mathrm{~N}_{2}, \mathrm{~N}_{3}$ & $18000,8000,4500$ & $18000,4500,980$ & $18000,4500,980$ \\
\hline $\mathrm{r}_{21}$ & 1.5 & 2.0 & 2.0 \\
\hline $\mathrm{r}_{32}$ & 1.333 & 2.143 & 2.143 \\
\hline$\phi_{1}$ & 6.063 & 10.7880 & 6.0042 \\
\hline$\phi_{2}$ & 5.972 & 10.7250 & 5.9624 \\
\hline$\phi_{3}$ & 5.863 & 10.6050 & 6.0909 \\
\hline $\mathrm{p}$ & 1.53 & 0.75 & 1.51 \\
\hline$\phi_{\mathrm{ext}}^{21}$ & 6.1685 & 10.8801 & 6.0269 \\
\hline $\mathrm{e}^{32}$ & $1.8 \%$ & $1.1 \%$ & $2.2 \%$ \\
\hline $\mathrm{e}_{\mathrm{a}}^{21}$ & $1.5 \%$ & $0.6 \%$ & $0.7 \%$ \\
\hline $\mathrm{e}_{\mathrm{ext}}^{21}$ & $1.7 \%$ & $0.9 \%$ & $0.4 \%$ \\
\hline $\mathrm{GCI}_{\mathrm{fine}}{ }^{21}$ & $2.2 \%$ & $1.1 \%$ & $0.5 \%$ \\
\hline
\end{tabular}

\subsubsection{Numerical Uncertainty and Discretization Error Bars}

Deduction of uncertainty from errors is not a trivial problem in the absence of statistical/random sampling. Equation (15d) is to be interpreted as an uncertainty estimate (Roache 1997, 1998) because of the explicit safety factor $F_{s}=1.25$. Eq. (15c) also involves a varying safety factor implicitly (see Celik \& Karatekin, 1997). Whereas, Eq. (15a\&b) express simply the value of the approximate relative error. For the calculation of a more conservative estimate uncertainty, we suggest the following estimate given by

$\bar{\delta}_{\text {dis }}=\operatorname{mean}\left(e_{a}^{32}, e_{a}^{21}, e_{e x t}^{21}, G C I^{21}\right)$

with the confidence interval given by

$$
(1-\alpha) \times 100 \% \text { C.I. of } \bar{\delta}_{d i s}=\left(\bar{\delta}_{d i s}+k_{d i s}, \bar{\delta}_{d i s}-k_{d i s}\right)
$$

where

$$
k_{d i s}=s_{d i s} t_{\alpha / 2}
$$

$s_{\text {dis }}$ is the point estimate of the standard deviation of all the values calculated from Eqs. $15 \mathrm{a}, \mathrm{b}$, c, and d and $t_{\alpha / 2}$ is calculated from the T-distribution with $v=\mathrm{n}-1=3$ degree of freedom. 
When computed profiles of a certain variable are presented, it is recommended that numerical uncertainty is indicated by error bars on the profile, analogous to experimental uncertainty. It is further recommended that this be done using the GCI in conjunction with an average value of $p=p_{\text {ave }}$ as a measure of the global order of accuracy. This is illustrated in Figs. 3 and 4.

Figure 3 (data taken from Celik \& Karatekin 1997) presents an axial velocity profile along y-axis at an axial location of $\mathrm{x} / \mathrm{H}=8.0$ for a turbulent two-dimensional backward-facing-step flow. The three sets of grids used were 980,4500 , and 18000 cells, respectively. The local order of accuracy $p$ calculated from Eq. (13) ranges from 0.012 to 8.47, with a global average $p_{\text {ave }}$ of 1.49 , which is a good indication of the hybrid method applied for that calculation. Oscillatory convergence occurs at $20 \%$ of the 22 points. This averaged apparent order of accuracy is used to assess the GCI indicies given by Eq. (15d) for individual grids, which is plotted in the form of error bars, as shown in Figure 3(b). The maximum discretization uncertainty is $10 \%$, which corresponds to $\pm 0.35 \mathrm{~m} / \mathrm{s}$.

Figure 4 (data taken from Celik \& Badeau 2005) presents an axial velocity profile along the y-axis at the station $\mathrm{x} / \mathrm{H}=8.0$ for a laminar two-dimensional backward-facing-step flow. The Reynolds number based on step height is 230 . The sets of grids used were $20 \times 20,40 \times 40$, and $80 \times 80$, respectively. The local order of accuracy $p$ ranges from 0.1 to 3.7 , with an average value of $p_{\text {ave }}=1.38$. In this figure, $80 \%$ out of 22 points exhibited oscillatory convergence. Discretization error bars are shown in Figure 3(b), along with the fine-grid solution. The maximum discretization error was about $100 \%$; this high value is relative to a velocity near zero, and corresponds to a maximum uncertainty in velocity of about $\pm 0.012 \mathrm{~m} / \mathrm{s}$
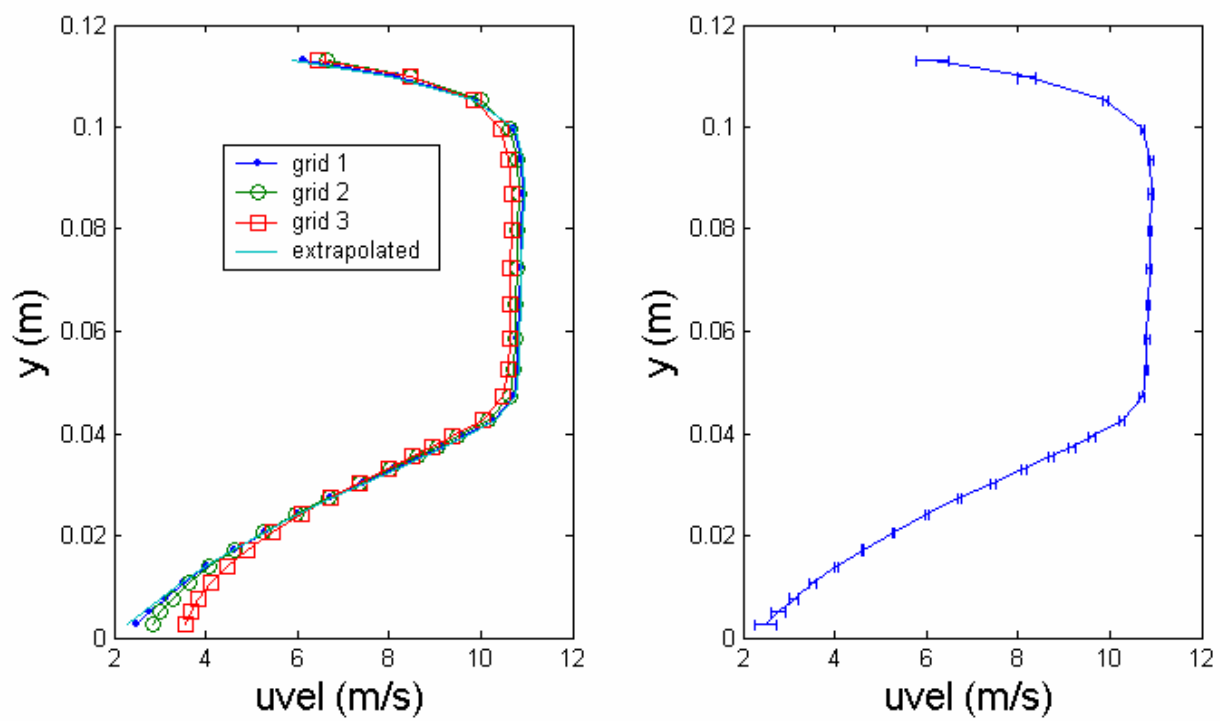

Figure 3. (a): Axial velocity profiles for a two-dimensional turbulent backward-facing-step flow calculation, Ref: Celik \& Karatekin [1997]; (b): Fine-grid solution, with discretization error bars computed using Eq. (15d). 

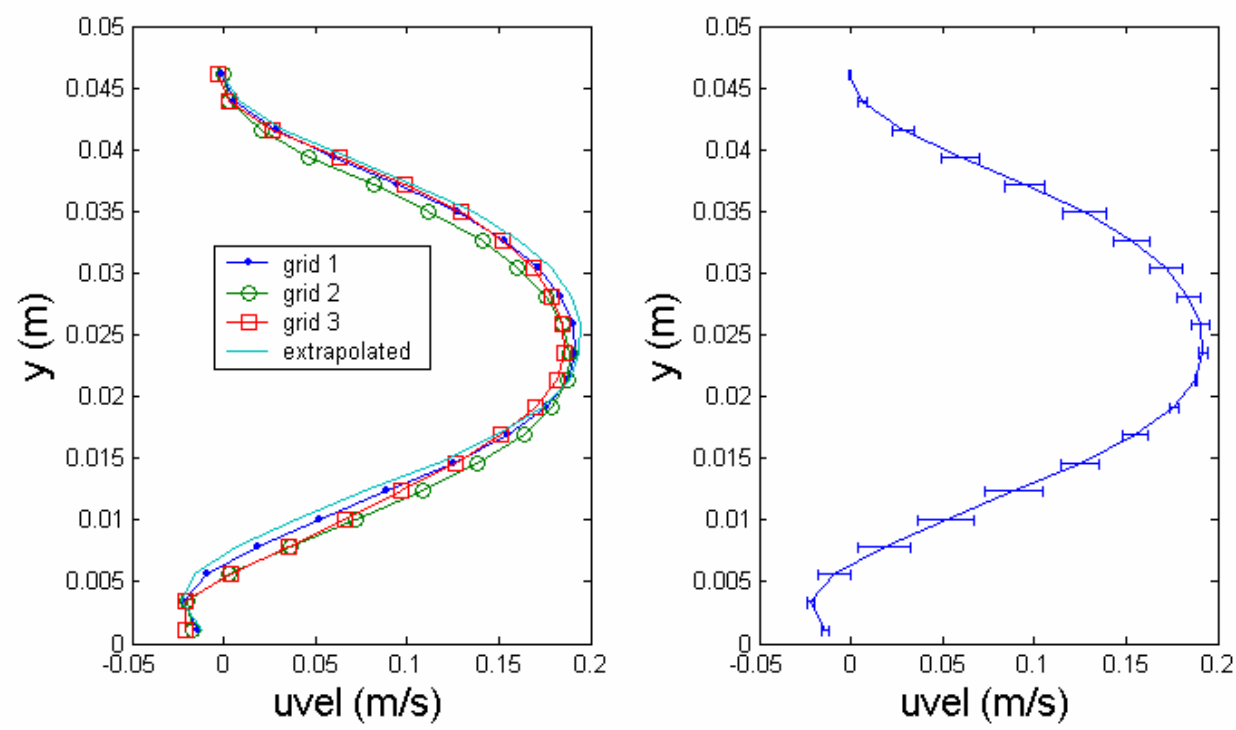

Figure 4. (a): Axial velocity profiles for a two-dimensional laminar backward-facing-step flow calculation, Ref: Celik \& Badeau [2005]; (b): Fine-grid solution, with discretization error bars computed using Eq. (15d).

\subsection{Calculation Validation}

Of the two prevalent conceptions of validation Roache [1998], the Problem Oversight Committee has adopted the view of validation exercise not necessarily including a pass-fail tolerance, but of simply establishing the level of validation. For example, at a naive level of comparison, if the comparison with experimental data shows a discrepancy of $10 \%$ then the calculation will be reported to have been validated to $10 \%$, regardless of the adequacy of this tolerance for the eventual application. Gross discrepancy will not be described as "validated" to any level.

\subsubsection{Method for Determination of Validation Level}

The validation level will be established with the following procedure, following Coleman [2003] modified to encompass $95 \%$ certainty level. This criterion is also consistent with the ASME Standard in the forthcoming ASME Guide for Verification and Validation in Computational Fluid Dynamics [2007]).

The experimental results (data) are denoted by $\mathrm{D}$, and the simulation results by $\mathrm{S}$. The comparison error $\mathrm{E}$ is defined as

$E=D-S$

The comparison error is the naive measure of validation, but it does not take into account the uncertainties in either the experimental data or the simulation. We replace this naive measure with the desired validation level $L v$ as follows.

The validation uncertainty $U_{\text {val }}$ is defined as the combination of all of the experimental uncertainties $U_{d}$, the uncertainty in the simulation result due to the uncertainties in the simulation inputs $\mathrm{U}_{\text {input }}$, and the uncertainty due to the numerical solution of the equations $\mathrm{U}_{\text {num. }}$. 
$U_{\text {val }}^{2}=U_{d}^{2}+U_{\text {num }}^{2}+U_{\text {input }}^{2}$

This validation uncertainty can be viewed as the noise level imposed by all of the uncertainties in the experimental values $\mathrm{D}$ and those uncertainties in the simulation $\mathrm{S}$ due to both the simulation inputs and the numerical solution of the equations. It is the estimated error bar for $\mathrm{E}$ that would be expected if the modeling error $=0$.

Using $U_{\text {val }}$ and $|E|$ (the absolute value of the comparison error $E$ ) as metrics in the validation process, if $|\mathrm{E}|>>\mathrm{U}_{\mathrm{val}}$ then the sign and magnitude of $\mathrm{E}$ can be taken as indicative of the modeling error, and the level of validation $L v$ is specified as $|\mathrm{E}|$. On the other hand if $|\mathrm{E}|<\mathrm{U}_{\mathrm{val}}$ or $|\mathrm{E}| \approx \mathrm{U}_{\mathrm{VAL}}$, then from an uncertainty point of view nothing definitive can be concluded about the sign or magnitude of the modeling error, and the level of validation $L v$ can only be specified as $U_{\text {val. }}$. The modeling error may in actuality be small, but this cannot be concluded from the hypothetical Validation exercise because the level of uncertainties in the experiments and/or the simulations are excessive, swamping the comparison error E. In summary, the validation level $L v$ will be given by

$$
L v=\max \left(|E|, U_{v a l}\right)
$$

The Validation exercise as described here will always produce some non-zero Level of Validation. However, the code/model cannot be claimed to be "validated" unless some reasonable level of agreement $\mathrm{E}$ is achieved in some useful metric (e.g. total heat transfer, maximum temperature, etc.) In addition to this validation metric, trends must be predicted to some reasonable level. Note that trends can be included in the basic definition by defining another metric as the sensitivity of the base metric to a parameter, e.g. $\Delta$ (max temperature $) / \Delta$ (flow rate) .

The remaining metric of importance is the level of validation required from a programmatic standpoint, $\mathrm{U}_{\text {reqd. }}$. The adequacy of the simulation will ultimately be determined by the requirement that the validation level $L v$ satisfies

$$
L v \leq U_{\text {reqd }}
$$

but contributors need not be concerned with this determination.

\subsubsection{Data Reporting Requirements for Standard Problem Participants}

Standard problems selected to support thermo hydraulic method development are expected to fall into three categories: 1) blind validation problems, 2) open validation problems, and 3) code-to-code benchmark comparison problems. In the first case, only experimental data needed to properly define boundary conditions will be released to problem participants, and only the oversight committee will have access to experimental data needed to calculate uncertainty levels. In the second case, the full data set will be available to all participants, allowing participants to calculate uncertainty levels prior to submission. In the third case, experimental data is unlikely to be available for true validation, and uncertainty levels calculated will reflect only the difference between submitted calculations and a reference calculation defined by the oversight committee. In this third case, no calculation will be described as "benchmarked" rather than "validated", but a comparable procedure will be employed.

For each standard problem, a set of validation parameters (e.g., velocity, pressure, or temperature) will be specified for calculation of error and validation level based on the phenomena of interest identified in each experiment. The spatial position and distribution (e.g., at a single point, along a line, or within a 
plane) of the validation measurement within the experimental geometry will also be specified. Although these characteristics of the data used for calculation of validation error and validation level may vary greatly from one standard problem to the next, all standard problem contributions must meet certain common criteria to allow consistent calculation of validation error and validation level for all contributions and to allow meaningful comparisons to be made between contributions.

\subsubsection{Boundary Conditions}

All experiments or benchmarks accepted and released as Standard Problems will include a detailed specification of all boundary conditions needed for calculation of the desired parameters. As confirmation that boundary conditions have been properly applied, all contributions must report values and/or distributions of each relevant parameter at each boundary which serves as a source or sink for the model. The error in the boundary condition distribution, particularly if distribution functions are used to approximate pointwise experimental data, should be assessed and reported. The error in the boundary conditions used in the model versus the experimental data provided in the problem specification as a consequence of problem nodalization should also be assessed and reported. As an example, the approximation of circular cylinder inlet by hexahedral or tetrahedral elements results in a slight reduction in cross-sectional flow area in the computational model. As a result, the total mass flux through the test sections might be underestimated, if boundary data is provided in the forma of an average velocity or time-averaged velocity distribution. Boundary condition errors resulting from such approximations should be quantified and reported.

The total conservation error over the computational domain for each of the continuity, momentum, energy, and, if applicable, scalar equations should also be reported for each submission.

\subsubsection{Numerical Convergence}

As a consequence of the wide variety of approaches which may be included as contributions to the Standard Problem exercises, the specification of a single mandatory methodology for the determination of calculation convergence in all contributions is impractical. Choice of convergence monitoring methodology will be left to each individual contributor. However, contributors must report the minimum acceptable convergence criteria based on the method employed as well as the convergence level achieved. It is recommended that CFD analysts employ the method for determining sensitivity to iterative convergence described in Section 3.3 and report the results.

\subsubsection{Turbulence}

Since turbulent flow is dominant in nearly all regions of interest in a VHTR system, it is anticipated that all Standard Problems will include regions of turbulent flow. Regardless of whether steady or unsteady methods for turbulence modeling are employed in the calculation or the experiment, the Reynolds averaging procedure will be employed in the calculation of validation error and validation level. Reynolds averaging assumes that any parameter can be represented as the sum of an average component $\overline{\mathrm{u}}$ and a fluctuating component $\mathrm{u}^{\prime}$.

$$
\mathrm{u}=\overline{\mathrm{u}}+\mathrm{u}^{\prime}
$$

Although $\bar{u}$ is named the average component, it need not necessarily be limited to constant values and the inclusion of low frequency fluctuations resulting from relatively large-scale periodic flow structures as part of $\bar{u}$ may actually be preferable for validation if suitable experimental data is available for comparison. As an example, a turbulent flow parameter signal is shown in Figure 5. Assuming that 
adequate experimental validation data is available, the decomposition of a signal into average and fluctuating components can be defined in any of the ways shown in Figure 6.

In general, all steady state solvers are expected to report a constant value for the average component as shown in Figure 6a. If an unsteady solver is employed, some large turbulence structures may be resolved by the unsteady simulation of the Navier-Stokes equations. Although the use of a constant value for the average component is generally preferred, data from such cases may be reported using definitions of the average component which are more consistent with Figures $6 \mathrm{~b}$ or $6 \mathrm{c}$. Therefore, when an unsteady solver is used, the contributor must define the methodology used to decompose reported values of the average component $\bar{u}$ and the fluctuating component $u^{\prime}$.

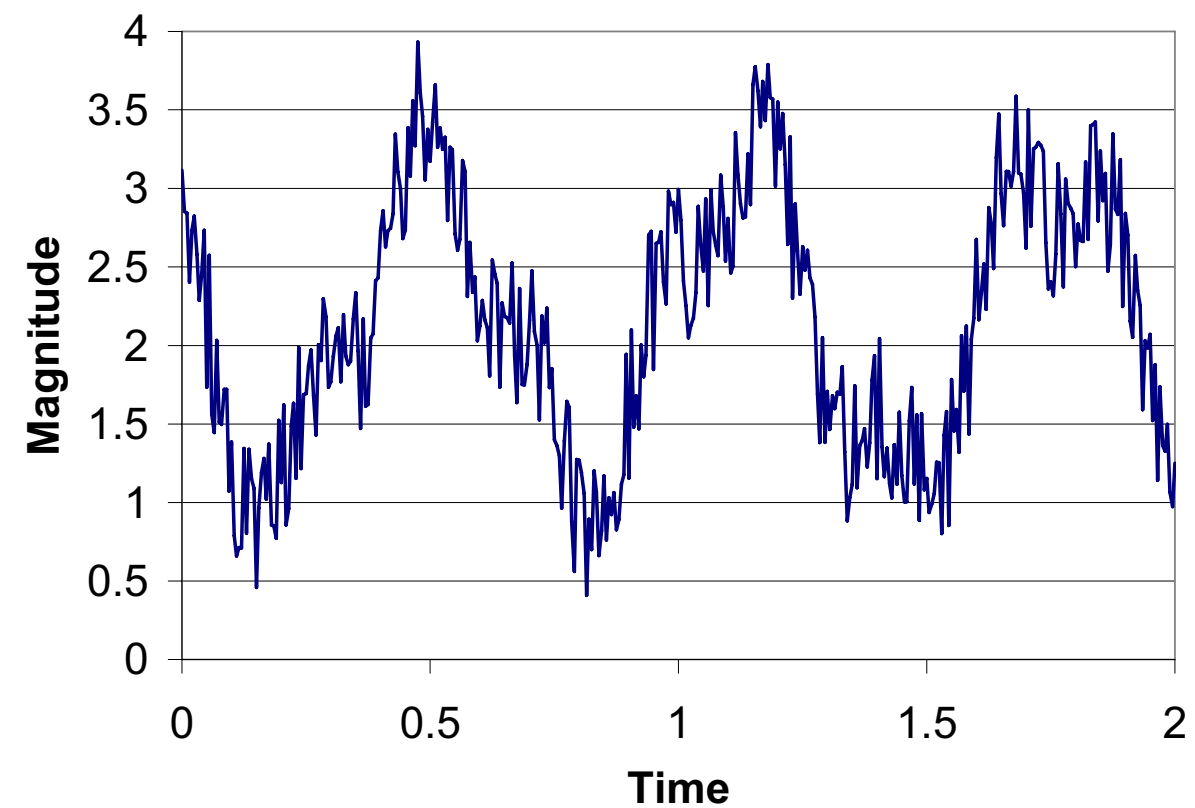

Figure 5. Sample signal from numerical simulation. 
$\bar{u}$

a)

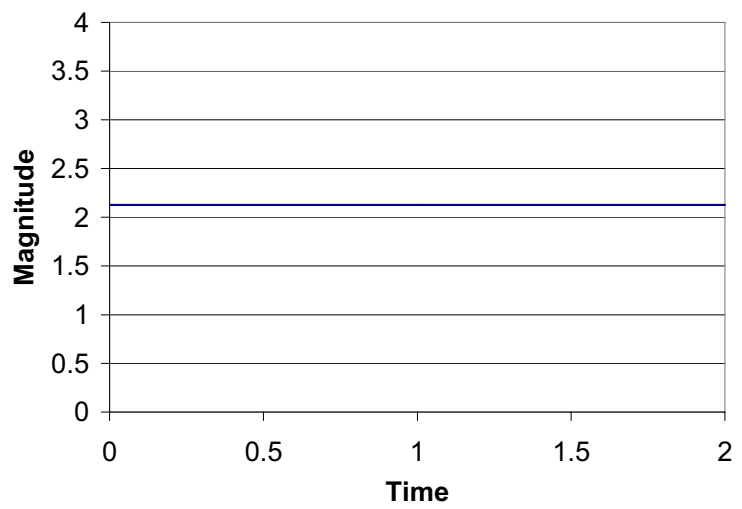

b)

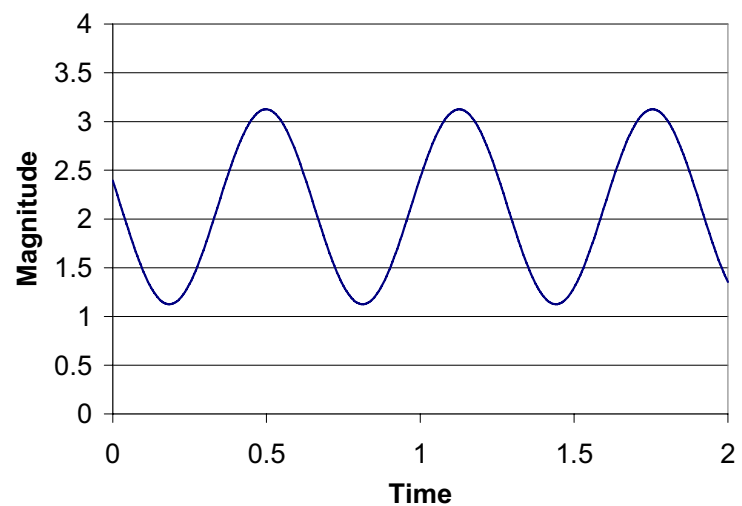

c)

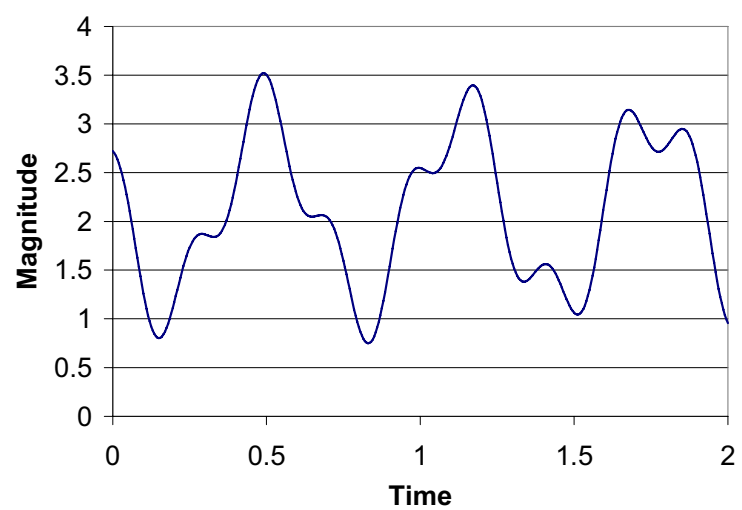

$u^{\prime}$
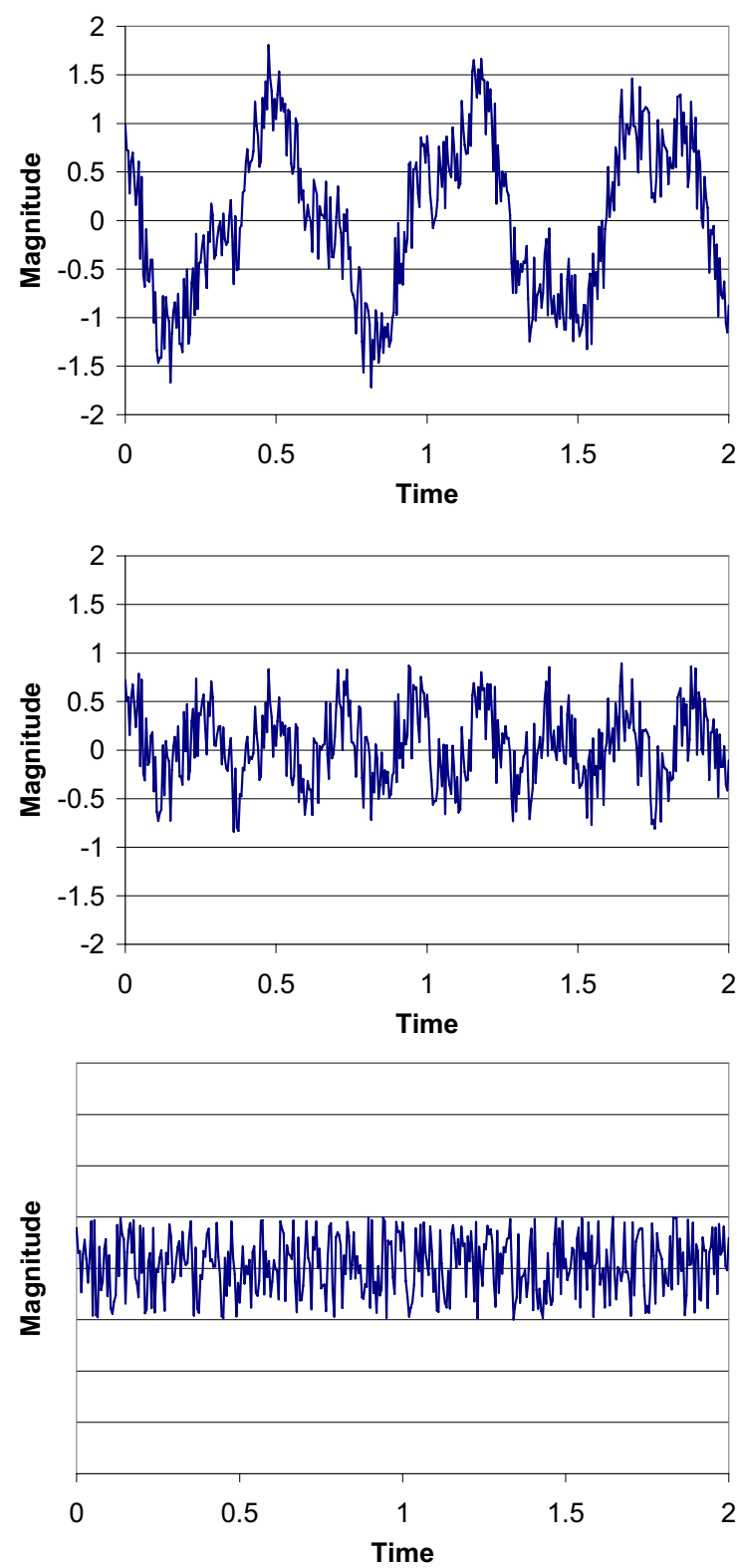

Figure 6. Three sample decompositions of the signal shown in Figure 5 into average, $\bar{u}$, and fluctuating, $u^{\prime}$, components. In (a), the average component has a constant value and all fluctuations are included in the fluctuation component. In (b), the primary periodic part of the fluctuation is included in the average component definition. In (c), the sum of the primary and secondary periodic parts of the fluctuations are included in the average component definition. All decompositions are valid applications of the Reynolds averaging methodology.

\subsection{Experimental Accuracy and Guidelines}

Flow measurements are required to increase our understanding of the physical phenomena in flowing systems, particularly in three-dimensional complex flows. The advancement in the development of computational fluid dynamics requires much experimental data and the measurement of many different 
flow parameters. These include the instantaneous velocity magnitudes, vector velocities themselves, spatial and temporal correlations, Reynolds shear stresses and full field three-dimensional velocity components. There is often a need for more complete and more accurate information than had been required before for CFD validation.

The clear understanding of the problem at hand to determine the type of instrumentation and the interrelated roles of the instrumentations is evident. The need and type of the measurements should be defined before undertaking the physical measurement. Most of the types of measurement techniques depend on the nature of the flow and the physical parameters needed, and these in turn govern instrument selection. Understanding the physical principles involved in the measurement is required since most of the measurements are indirect. For example, when a pressure is measured with a pressure transducer, the deflection of a membrane or diaphragm is used to determine the pressure. Hot-wire instrumentation is dependent on the physical laws relating heat transfer with flow velocity. Understanding the physical phenomena of the instrument helps in evaluation of the uncertainty and the measurement errors.

Various common measurements techniques are available such as:

1. pressure measurements
a. static pressure
b. total pressure
c. unsteady pressure measurements (depends on frequency response)
d. pressure sensitive paint (PSP) technique (based on oxygen-quenched photoluminescence).

2. temperature measurements
a. thermocouples
b. $\quad$ surface measurements devices (thin film, liquid crystals)
c. infrared thermography

3. hot wire and hot film anemometry

4. optical measurement techniques
a. Laser Doppler Velocimetry (LDV)
b. Particle Image Velocimetry (PIV)
c. Holography

The selection of the experimental techniques is based on the requirements, test apparatus, parameter selection, cost and time. Each experimental technique has its own advantages and disadvantages.

In order to tackle the issue of the experimental uncertainty, it is necessary to first define types of errors, which impact the experimental results. It is then required to list the most promising strategies in order to reduce or avoid errors. Based on these strategies, procedures have to be defined, which are used 
when making the measurements. For this purpose guidelines will be given in the future, obtained from various sources to provide a framework for proposed instrumentation.

In performing an experiment, several steps can be followed: 1) equipment identification, 2) test section design, fabrication and instrumentation: this involves setting up parameters to be studied and under what range of conditions, 3) flow measurements: this involves accurate measurements of the flow rate, pressure measurement devices, other measurement techniques, 4) test facility calibration, 5) data reduction and uncertainty analysis and 6) development of results and conclusions.

In most cases the experimenter tries to eliminate or reduce the errors from the results. As is well known, this goal is a difficult one. Consequently, the experimenter should provide the reader with some measure of the reliability of the data. There are two categories of experiment- single sample and multiple sample experiments. Ideally, the repetition of all measurements enough times using enough observers and enough diverse instruments can be performed so that the reliability can be assured by using statistics. Experiments in which uncertainties are evaluated by such repetition are called multiple sample experiments. Often, in most engineering experiments, it is not practical to estimate all of the uncertainties of observation by repetition and using diverse measurement devices. Most of the measurements are obtained by single-sample experiments.

The uncertainty is the difference between the true and observed values. These values depend upon the particular circumstances of the observation. Propagation of uncertainty means the way in which uncertainties in the various variables affect the uncertainty in the results. "Variable" is a basic quantity observed directly in the laboratory as opposed to the result which is obtained by making corrections to or calculations with the recorded values of the variables. The recorded values of the variables are known as data.

In order to arrive at a rational method for describing the uncertainties in the variables, it is necessary to discuss the sources and nature of the uncertainties. There are many types of errors which can contribute to the uncertainty in each variable. One classification of these errors is as follows: Accidental errors, fixed errors and mistakes. Accidental errors are those varying errors which cause repeated readings to differ without apparent reason. They arise from instrument malfunction and time lag, personal errors and many other causes. Fixed errors are those which cause repeated readings to be in error with the same amount. Mistakes are erroneous readings of scales, recorded wrong data.

A good error estimator for the data should have the following properties: 1) the point estimator should be unbiased, and 2) an unbiased estimator should have minimum variance. This property indicates that the minimum variance point estimator has a variance that is smaller than the variance of any other estimator of this parameter. Possible error sources must be examined to obtain overall uncertainty. Particular error sources are a function of the instrumentation used. A discussion of error sources for a particular method for velocity measurement is provided next.

\subsubsection{Velocity Measurement Techniques of the Standard Problems}

Digital particle tracking velocimetry techniques are being used in the standard problems test cases. This technique is a valuable tool capable of measuring the significant spatial structure of turbulent flows. Other experimental velocity instruments have relied on point measurement techniques-laser Doppler anemometry (LDV) and hot wire velocimetry, while providing high temporal resolution, these methods have limited spatial resolution. Laser Doppler anemometry and hot wire techniques have firmly established as standard tools in experimental fluid dynamics for some years already. However, particle image velocimetry (PIV) is a new scheme and is still progressing to be considered as such. However, until 
now there is no general documented best practice and guidelines in determining experimental uncertainty in employing these techniques.

A typical PIV set up is shown in Figure 7. It consists of laser illumination sources (such as two double oscillators ND:YAG lasers). The lasers are pulsed at various repetition rates. A camera is used to capture the images of the scattered light from the tracer seeds.

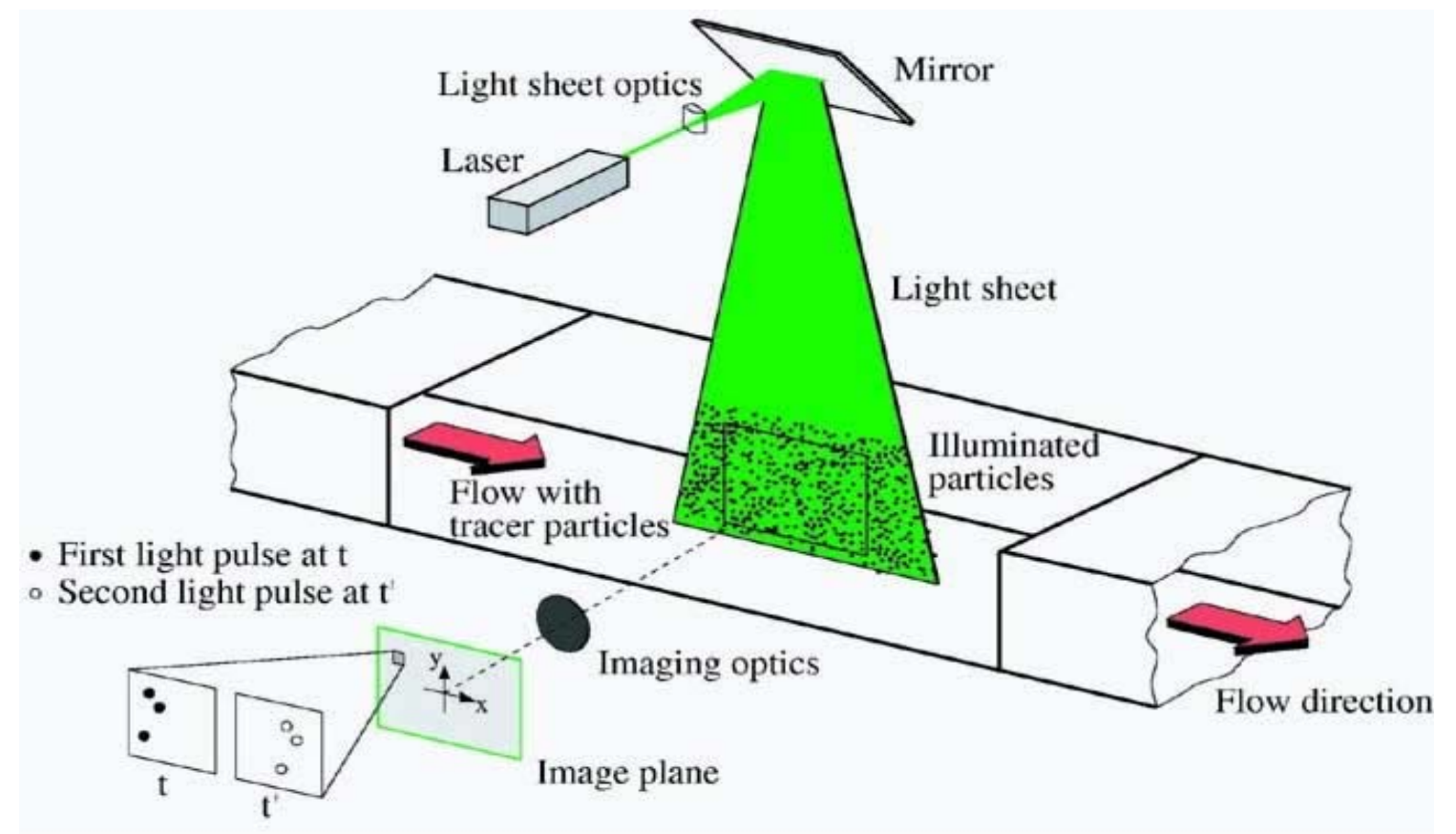

Figure 7. Typical Particle Image Velocimetry (PIV) Setup.

1. Triggering and system synchronization of the cameras with the laser pulses. The time spacing between the pulses and pulse width should also be selected depending on the flow velocity values to capture the flow fluctuations and it gradients. Figure 8 shows an example of a camera and laser synchronization scheme. 


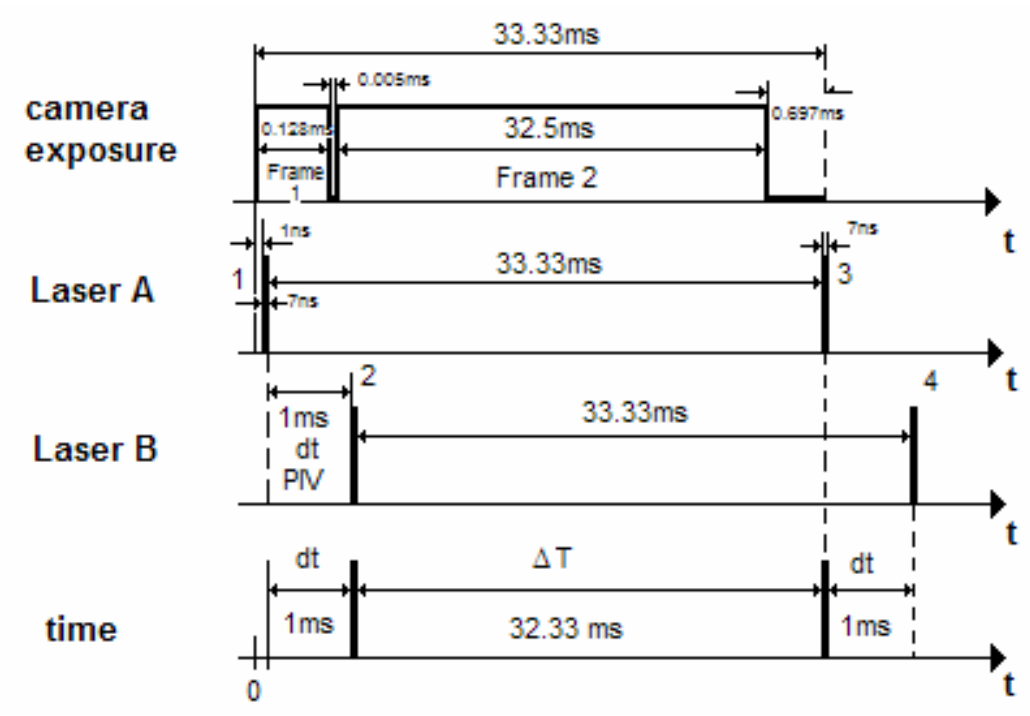

Figure 8. Synchronization between laser system and CCD camera.

2. The bias in velocity measurements due to the particle tracer size. PIV is a tracer technique and, as such, relies on the velocity information from small tracer particles contained in and transported with the flow that is investigated. Only if the tracer particles faithfully follow any changes of the velocity can one expect the measurements to yield velocity data which accurately represent actual flow velocity. If the tracer particles are too large or their density is too high then, as a result of their inertia, they may not respond to velocity changes sufficiently and may display a velocity lag instead. This, in turn, results in the collection of experimental velocity data which do not represent actual flow. In the measurement of turbulence fields, the maximum frequency that particles should be able to follow can be quantified based on the Kolmogorov scale. The particle response to step acceleration can be obtained from the relaxation time which depends on the density and size of the particle.

3. Tracking routine errors due to out-of-plane motions, interpolation errors, etc. These tracking routines should be evaluated. One of the difficulties is the determination of the world coordinate of the seeds which require calibration. Uncertainty in the seed location should be estimated. The task becomes more complicated in employing stereo particle image velocimetry. The location and displacement uncertainty estimations of the tracer should be determined. To improve the resolution and accuracy of the CCD an algorithm capable of tracking displacements to sub-pixel accuracy could be employed. A typical setup for stereo particle image is illustrated in Figure 9.

4. Interpolation errors obtained from interpolating randomly located PTV vectors onto a uniform grid in order to calculate turbulence statistics. 


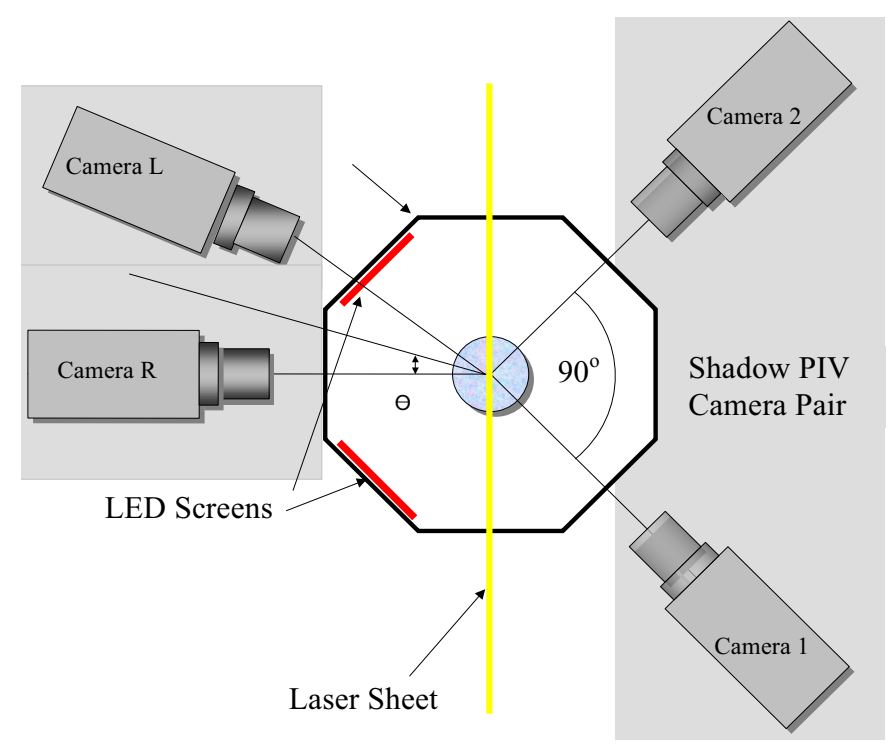

Figure 9. PIV and SPIV camera arrangement for 3-D two-phase bubbly flow measurements.

\subsubsection{Policy Statements on Experimental Accuracy}

This section contains the policy statements on experimental accuracy from two technical journals: 1) AIAA Journal 2) Journal of Fluids Engineering. At this point, it is recommended that these guidelines be followed by experimentalists who intend to provide data having Standard Problem quality. 


\section{AIAA Journal Editorial Policy Statement on Experimental Accuracy}

\section{Experimental Accuracy}

The accuracy of experimental results is concerned with how well the specified measurands in the study have been determined, and the statistical confidence with which they can be assessed. The appropriateness of the measurands for describing the physical phenomena and for comparing the results with numerical investigations is not part of this policy statement. The intent of the policy statement is to encourage authors to provide sufficient information for readers to independently assess the statistical confidence of results presented in AIAA journal articles. Authors should address the following criteria, as applicable, in summary form in the manuscript and can also provide additional detailed information in referenced documents:

1. Statement of Measurement Techniques and Apparatus: Authors should be clear and precise in the description of the test articles and facilities, test methods, and instrumentation, including the data reduction techniques.

2. Statement of Experimental Design: To assist others in assessing the uncertainty in the experimental results, for such purposes as reproducing them experimentally or simulating them analytically and for identifying corresponding statistical techniques to analyze the results, the experimental conditions and order of acquisition should be presented or concisely described.

3. Estimation of Uncertainty: Unexplained variance in results should be addressed as follows:

a. Estimation of Bias Uncertainty: The bias uncertainty of the results (systematic component of the unexplained variance) should be estimated. In addition, the method of estimation should be described.

b. Estimation of Precision Uncertainty: The repeatability of the results (random component of the unexplained variance) should be quantified. Also, the method of determining the repeatability should be specified. Unusual results, whether explained or unexplained, should be identified.

c. Statement of Total Uncertainty: The total uncertainty is the combination of the bias and precision uncertainties and should be presented with the experimental results (in text, tables, or graphs as appropriate). Authors should identify any known potential sources of bias and precision uncertainty that have not been explicitly included in the estimates of total uncertainty.

4. Statistical Analysis: Statistical analysis may be warranted in a number of cases, such as when comparing the effects of independent variables, when correlating experimental conditions and measures, and when performing hypothesis tests.

a. Description of Methodology: The choice of statistical analysis method should be clearly described and justified relative to a priori knowledge, the experimental design, and the characteristics of the measurements.

b. Treatment of Impact: The findings of the statistical tests should be carefully substantiated and discussed relative to their confidence level, parametric assumptions, and experiment design.

5. Coverage Factor: The coverage factor used for all expanded uncertainty estimates should be specified. That is, the author(s) should associate a specific level of confidence with each uncertainty value, by estimating the probability that reported error limits will encompass the true result.

For more detailed discussion of the above topics, prospective authors should consult modern texts in measurement methods, experimental design, uncertainty analysis, hypothesis testing, and statistical data analysis. 


\section{ASME Journal of Fluids Engineering September 1991 Editorial on Experimental Uncertainty}

In 1987, the Coordinating Group for Fluid Measurements (CGFM) of the Fluids Engineering Division (FED) was asked by Dr. Frank White, the Technical Editor of the Journal of Fluids Engineering (JFE) at that time, to prepare a set of guidelines on estimating experimental uncertainty. The purpose was to alert the authors of the Journal to the fact that estimates of experimental uncertainty enhance the value of information reported. It was also felt that the publication of such guidelines would improve the uniformity of presenting experimental data in the pages of the Journal. Many members of the Committee felt at that time that other reasons justified the publication of such guidelines, as for example, the need for authors to differentiate between bias and precision error and the need to handle correctly single-sample experiments.

The CGFM reviewed existing standards, including PTC19.1 and the material presented in a collection of papers from JFE in 1985. There is no question that the basic information on how to handle uncertainty is already published. However, it is not written in a practical format as evidenced by usage (or the lack thereof). Existing information is in articles that are too long, depend too much on multiple sample analysis, do not provide perception on how to handle bias error, and give the impression that uncertainty analysis re-quires disproportionate attention. The current stat4ement in JFE refers authors to those articles but leaves the actual reporting format up to each author without stringent requirements.

Over the past two years the CGFM has struggled to reach a consensus agreement on this statement. A perception has evolved that there must necessarily be three steps to develop good practices in reporting uncertainty estimates. First, a broad outline of policy must be introduced to recognize bias and precision error and the limits for the uncertainty band. Second, terminology must be standardized. Much of the problem in communicating in-formation about uncertainty is in the language. The particular problem is that a differentiation between single and multiple sample experiments in the context of the notion that these are but endpoints on a continuum must be made. This seems simple enough, but it is incredibly difficult to accomplish. Third, procedures for handling error, and especially bias error, need to be standardized. So far this seems only possible by using examples.

CGFM intends to continue the steps outlined above, and considers the first step as having been completed with the publication of the following guidelines. These guidelines were arrived at after long discussions and exchange of arguments between the CGFM, some technical associate editors of the Journal, some reviewers and the Technical Editor. Special appreciation is extended to H. W. Coleman and W. G. Steele, the principal authors of the adopted statement.

Edwin P. Rood

Chairman, Coordinating Group for Fluid Measurements

Demetri P. Telionis

Technical Editor, Journal of Fluids Engineering 


\section{Journal of Fluids Engineering Policy on Reporting Uncertainties in Experimental Measurements \& Results GUIDELINES}

An uncertainty analysis of experimental measurements is necessary for the results to be used to their fullest value. Authors submitting papers for publication to this Journal are expected to describe the uncertainties in their experimental measurements and in the results calculated from those measurements. The presentation of experimental data should include the following information:

1) The precision limit, $P$. The $\pm P$ interval about a result (single or averaged) is the experimenter's 95 percent confidence estimate of the band within which the mean of many such results would fall, if the experiment were repeated many times under the same conditions and using the same equipment. The precision limit is thus an estimate of the scatter (or lack of repeatability) caused by random errors and unsteadiness.

2) The bias limit, $B$. The bias limit is an estimate of the magnitude of the fixed, constant error. When the true bias error in a result is defined as $\beta$, the quantity $B$ is the experimenter's 95 percent confidence estimate such that $|\beta| \leq B$.

3) The uncertainty $U$. The $\pm U$ interval about the result is the band within which the experimenter is 95 percent confident the true value of the result lies. The 95 percent confidence uncertainty is calculated from

4) A brief description of, or reference to, the methods used for the uncertainty analysis. (If estimates are made at a confidence level other than 95 percent, adequate explanation of the techniques used must be provided.) The estimate of precision limits and bias limits should be made corresponding to a time interval appropriate to the experiment.

It is preferred that the following additional information also be included:

1) The precision limit and bias limits for the variables and parameters used in calculating each result.

2) A statement comparing the observed scatter in results on repeated trials (if performed) with the expected scatter $( \pm \mathrm{P})$ based on the uncertainty analysis. Although it is natural in any experimental paper to discuss sources of experimental error in the body of the text, this alone does not satisfy our requirement. All reported data must show uncertainty estimates. All tables should carry estimates. All figures reporting new data should contain uncertainty estimates either on the figure itself or in the caption. 1. A list of references on the topic, many of which appeared in the pages of this Journal is provided here in alphabetical order.

A list of references on the topic, many of which appeared in the pages of this Journal is provided here in alphabetical order.

\section{EXAMPLE}

Consider an experiment in which the pressure drop characteristics for fully developed flow conditions in a particular type of circular pipe are determined over a range of water flow rates. The outcome of this experiment might be presented by plotting one result — the Fanning friction factor, $f$, versus another result, the Reynolds number, Re. To obtain each "data point" that would be plotted on such a figure, the values of $f$ and Re could be calculated from 


$$
\begin{aligned}
& f=\frac{\pi^{2}}{32} \frac{D^{5}}{\rho Q^{2}} \frac{\left(p_{1}-p_{2}\right)}{\left(x_{2}-x_{1}\right)} \\
& \operatorname{Re}=\frac{4}{\pi} \frac{\rho Q}{\mu D}
\end{aligned}
$$

where $Q$ is the volumetric flow rate of the water with density $\rho$ and dynamic viscosity $\mu, D$ is the pipe diameter, $\mathrm{p}$ is the static pressure, $\mathrm{x}$ is axial position along the pipe, and the subscripts 1 and 2 refer to the upstream and down-stream pressure tap locations, respectively.

The measured variables $(\mathrm{Q}, \mathrm{D}, \mathrm{p} 1, \mathrm{p} 2, \mathrm{x} 1, \mathrm{x} 2)$ and the parameters found from reference property data $(\rho, \mu)$ contain bias errors and precision errors. For example calibrating pressure transducers under static conditions may later introduce bias errors if the measured field involves dynamic motions. Other bias errors arise from calibration of the measurement systems for $\mathrm{p}$ and $\mathrm{Q}$ against imperfect standards and from using property values originally deter-mined in imperfect experiments. Precision errors could arise, for example, from sensitivity of the pressure transducer, flowmeter and data acquisition system to variations in ambient temperature and humidity. Inability to hold flow rate exactly constant during a period of data acquisition could also appear as a variation in the pressure measurements.

Errors in these quantities will propagate through Eqs. (2) and (3) to produce bias and precision errors in the results $f$ and Re. The techniques of uncertainty analysis described in the references can be used to obtain estimates of the bias limits and precision limits for the variables and parameters and the bias limit, $\mathrm{B}$, the precision limit, $\mathrm{P}$, and the uncertainty, $\mathrm{U}$, in the quantities $f$ and Re.

If the two pressures, $\mathrm{p} 1$ and $\mathrm{p} 2$ are measured successively using the same absolute pressure transducer, the bias errors in the measurements of the two variables will not be independent of each other. This phenomenon of correlated bias errors occurs fairly often in the fluid and thermal sciences, usually when variables are measured using the same transducer or using different transducers that have been calibrated against the same standard. These effects must be taken into account in the uncertainty analysis. A method for doing this is shown in one example in ANSI/ASME PTC 19.1 and is derived and discussed in detail in Chapter 4 of Coleman and Steele (1989). 


\section{REFERENCES}

Abernethy, R. B., Benedict, R. P., and Dowdell, R. B., 1985, “ASME Measurement Uncertainty,” ASME JOURNAL OF FLUIDS ENGINEERING, 107, pp. 161-164.

ASME, Guide for Verification and Validation in Computational Fluid Dynamics, ASME Committee V\&V 20, to appear in 2007.

ASME Journal of Fluids Engineering, 144, June 1996, 427.

Celik I, Karatekin O. "Numerical experiments on application of Richardson extrapolation with nonuniform grids". ASME Journal of Fluids Engineering 1997; 119:584 -590.

Celik I, Li J, Hu G, Shaffer C. "Limitations of Richardson extrapolation and some possible remedies". ASME Journal of Fluids Engineering 2005; 127:795 -805.

Celik I, Li J. "Assessment of numerical uncertainty for the calculations of turbulent flow over a backward-facing step". International Journal for Numerical Methods in Fluids 2005; 49:10151031.

Celik, I. and Badeau, A. Jr. "Verification and Validation of DREAM Code," Report No. MAE_IC03/TR103, Mechanical and Aerospace Engineering Department, West Virginia University, Morgantown, WV, 2005.

Celik, I., Chen, C.J., Roache, P.J. and Scheurer, G. Editors. "Quantification of Uncertainty in Computational Fluid Dynamics,” ASME Publ. No. FED-Vol. 158, ASME Fluids Engineering Division Summer Meeting, Washington, DC, 20-24 June, 1993.

Celik, I. and Hu, G., "Single Grid Error Estimation Using Error Transport Equation", ASME Journal of Fluids Engineering, 2004; 126:778-790.

Celik, I., Klein, M., Freitag, M. and Janicka, J. “Assessment Measures for URANS/DES/LES: An Overview with Applications," Journal of Turbulence 2006b; 7: 1-27.

Coleman, H. W., Some Observations on Uncertainties and the Verification and Validation of Simulations, ASME Journal of Fluids Engineering, Vol. 125, No. 4, pp. 733-735, July 2003.

Coleman, H. W., and Steele, W. G., Experimentation and Uncertainty Analysis for Engineers, John Wiley \& Sons, New York, 1989.

Coleman, H. W., and Steele, W. G., Experimentation and Uncertainty Analysis for Engineers, John Wiley \& Sons, New York, 1999.

Eça, L. and Hoekstra, M. "An Evaluation of Verification Procedures for CFD Applications," 24th Symposium on Naval Hydrodynamics, Fukuoka, Japan, 8-13 July, 2002.

Eça L, Hoekstra, M. (eds). Proceedings of the First Workshop on CFD Uncertainty Analysis, Lisbon, 2122 October, 2004a.

Eça L, Hoekstra M. (2004b) "On the Grid Sensitivity of the Wall Boundary Condition of the k- $\omega$ Turbulence Model,” ASME Journal of Fluids Engineering, 126, 900-910 
Eça L, Hoekstra, M. (eds). Proceedings of the 2nd Workshop on CFD Uncertainty Analysis, Lisbon, 2223 October, 2006a.

Eça, L. and Hoekstra, M. "On the Influence of the Iterative Error in the Numerical Uncertainty of Ship Viscous Flow Calculations," 26th Symposium on Naval Hydrodynamics, Rome, Italy, 17-22 September, $2006 b$.

Eça, L., Hoekstra, M., Hay, A. and Pelletier, D. "On the Construction of Manufactured Solutions for One and Two-Equation Eddy-Viscosity Models," Submitted for Publication to International Journal of Numerical Methods in Fluids, July 2006.

Elizalde-Blancas, F. and Celik, I. "Verification of various uncertainly measures using manufactured solutions", 2006.

Elizalde-Blancas F., Celik I., Pakalapati S. "Uncertainty Estimation for Numerical Solutions of Wall Bounded Turbulent Flows," Proceedings of the 2006 ASME Joint U.S.-European Fluids Engineering Summer Meeting, Miami, FL., USA, 17-20 July, 2006.

Ferziger, J.H., 1989 "Estimation and Reduction of Numerical Error," ASME Winter Annual Meeting, San Fransisco, December, 1989

Ferziger, J.H., Peric, M. (1996) "Further Discussion of Numerical Errors in CFD," International Journal for Numerical Methods in Fluids 1989; 23: 1263-1274.

Freitas, C. J., U. Ghia, I. Celik, H. Coleman, P. Raad, and P. J. Roache (2003), “ASME'S Quest to Quantify Numerical Uncertainty," AIAA Paper 2003-0627, AIAA 41st Aerospace Sciences Meeting, January 2003, Reno, Nevada.

Huebsch, W.W. "Two-Dimensional Simulation of Dynamic Surface Roughness for Aerodynamic Flow Control," accepted for publication in AIAA Journal of Aircraft, May, 2005

IAEA draft document, "Uncertainty Evaluation in Best Estimate Safety Analysis for Nuclear Power Plants."

Johnson, R.W. "Validation Studies for Numerical Simulations of Flow Phenomena Expected in the Lower Plenum of a Prismatic VHTR Reference Design," INL External Report INL/EXT-05-00787, September 2005.

Kline, S. J., and McClintock, F. A., 1953, "Describing Uncertainties in Single-Sample Experiments," Mechanical Engineering, 75, pp. 3-8.

Kline, S. J., 1985, “1983 Symposium on Uncertainty Analysis closure,” ASME JOURNAL OF FLUIDS ENGINEERING, 107, pp. 181-182.

Kline, S. J., "The Purposes of Uncertainty Analysis," ASME JOURNAL OF FLUIDS ENGINEERING, 107, pp. 153-160.

Lassahn, G. D., 1985, “Uncertainty Definition,” ASME JOURNAL OF FLUIDS ENGINEERING, 107, p. 179.

MacDonald, P. E., J. W. Sterbentz, R. L. Sant, P. D. Bayless, R. R. Schultz, H. D. Gougar, R. L. Moore, A. M. Ougouag, and W. K. Terry, NGNP Preliminary Point Design, Results of the Initial 
Neutronics and Thermal-hydraulic Assessments. Tech. Report INEEL/EXT-03-00870 Rev. 1, INEEL.

Measurement Uncertainty, ANSI/ASME PTC 19.1-1985 Part 1, 1986.

Menter, F., CFD Best Practice Guidelines for CFD Code Validation for Reactor-Safety Application, EVOL-ECORA-D01, February 2002.

Moffat, R. J., 1982, "Contributions to the Theory of Single-Sample Uncertainty Analysis," ASME JOURNAL OF FLUIDS ENGINEERING, 104, pp. 250-260.

Moffat, R. J., 1985, “Using Uncertainty Analysis in the Planning of an Experiment," ASME JOURNAL OF FLUIDS ENGINEERING, 107, pp. 173-178.

Moffat, R. J., 1988, "Describing the Uncertainties in Experimental Results," Experimental Thermal and Fluid Science, 1, pp. 3-17.

Orozco, C., Claramunt, K., Consul, R., and Oliva, A., "Finite Volume Computation and Verification of Fluid Flow and Heat Transfer in Domains with Moving Boundaries," European Congress on Comp. Meth. Appl. Sci. Eng., Jyvaskyla, Finland, July 24-28, 2004.

Pettersson-Reif, B. A., Gatski, T. B, and Rumsey, C. L., "On the behavior of two-equation models in nonequilibrium homogeneous turbulence,", Physics of Fluids, Vol. 18, 065109, 2006.

Richardson, L.F. "The Approximate Arithmetical Solution by Finite Differences of Physical Problems Involving Differential Equations, with an Application to the Stresses In a Masonary Dam," Transactions of the Royal Society of London, Ser. A 1910; 210: 307-357.

Richardson L.F. and Gaunt, J. A. "The Deferred Approach to the Limit," Philos. Trans. R. Soc. London Ser. A 1927; 226: 299-361.

Roache, P.J. "Code Verification by the Method of Manufactured Solutions," Journal of Fluids Engineering 2002; 124: 4-10.

Roache, P. J., Verification and Validation in Computational Science and Engineering, Hermosa Publishers, Albuquerque, 1998.

Roache, P.J. "Quantification of Uncertainty in Computational Fluid Dynamics," Annual Review of Fluid Mechanics 1997; 29: 123-160.

Roache, P.J. "A Method for Uniform Reporting of Grid Refinement Studies," Proc. Of Quantification of Uncertainty in Computation Fluid dynamics, Edited by Celik, et al., ASME Fluids Engineering Division Spring Meeting, Washington D.C., June 23-24, 1993, ASME Publ. No. FED-Vol. 158.

Roache, P.J., Ghia, K.N., White, F.M. "Editorial Policy Statement on Control of Numerical Accuracy,” ASME Journal of Fluids Engineering, 1986, Vol. 108, Mach Issue.

Rumsey, C. L., Pettersson-Reif, B. A., and Gatski, T. B., “Arbitrary Steady-State Solutions with the K-e Model,” AIAA Journal, Vol. 44, No. 7, July 2006, pp. 1586-1592. 
Simonin and Barcouda "Flow Through Staggered Tube Bundle" Data from ERCOFTAC data base found at http://cfd.me.umist.ac.uk/ercoftac/, flow case: C.78, 1986.

Smith, R. E., Jr., and Wehofer, S., 1985, "From Measurement Uncertainty to Measurement Communications, Credibility, and Cost Control in Propulsion Ground Test Facilities," ASME JOURNAL OF FLUIDS ENGINEERING, 107, pp. 165-172.

Stern, F., Wilson, R. V., Coleman, H. W., and Paterson, E. G. "Comprehensive Approach to Verification and Validation of CFD Simulations - Part 1: Methodology and Procedures," ASME Journal of Fluids Engineering 2001; 123: 793-802.

Stern, F., Wilson, R. and Shao, J. "Quantitative V\&V of CFD Simulations and Certification of CFD Codes,” International Journal for Numerical Methods in Fluids 2006; 50: 1335-1355.

Wilks, S. S., Determination of sample sizes for setting tolerance limits; Ann. Math. Statist. 12 (1941) 91-96.

Wilks, S.S., Statistical prediction with special reference to the problem of tolerance limits, Ann. Math. Statist. 13 (1942) 400-409. 